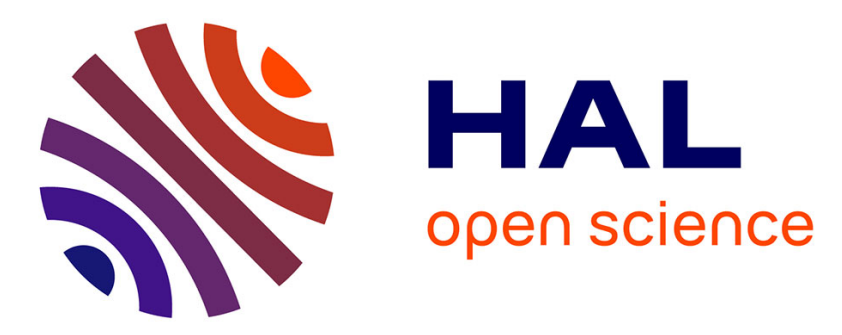

\title{
Ene reactions of 2-borylated $\alpha$-methylstyrenes a practical route to 4-methylenechromanes and derivatives
}

Chaima Boureghda, Aurélie Macé, Fabienne Berrée, Thierry Roisnel, Abdelmadjid Debache, Bertrand Carboni

\section{- To cite this version:}

Chaima Boureghda, Aurélie Macé, Fabienne Berrée, Thierry Roisnel, Abdelmadjid Debache, et al.. Ene reactions of 2-borylated $\alpha$-methylstyrenes a practical route to 4 -methylenechromanes and derivatives. Organic \& Biomolecular Chemistry, 2019, 17 (23), pp.5789-5800. 10.1039/c9ob00963a . hal02150447

HAL Id: hal-02150447

https://hal-univ-rennes1.archives-ouvertes.fr/hal-02150447

Submitted on 18 Nov 2019

HAL is a multi-disciplinary open access archive for the deposit and dissemination of scientific research documents, whether they are published or not. The documents may come from teaching and research institutions in France or abroad, or from public or private research centers.
L'archive ouverte pluridisciplinaire HAL, est destinée au dépôt et à la diffusion de documents scientifiques de niveau recherche, publiés ou non, émanant des établissements d'enseignement et de recherche français ou étrangers, des laboratoires publics ou privés. 


\section{Ene reactions of 2-borylated $\alpha$-methylstyrenes: a practical route to 4-methylenechromanes and derivatives}

Chaima Boureghda, ${ }^{\mathrm{a}, \mathrm{b}}$ Aurélie Macé, ${ }^{\mathrm{a}}$ Fabienne Berrée, ${ }^{\mathrm{a}}$ Thierry Roisnel, ${ }^{\mathrm{a}}$ Abdelmadjid Debache, ${ }^{\mathrm{b} *}$ and Bertrand Carboni ${ }^{a^{*}}$

4-Methylenechromanes were prepared via a three-step process from 2-borylated $\alpha$ methylstyrenes. This sequence is based on a key glyoxylate-ene reaction catalyzed by scandium(III) triflate. The resulting $\gamma$-hydroxy boronates, which cyclise to sevenmembered homologues of benzoxaborole on silica gel, were cleanly oxidized with sodium perborate, then cyclised under Mitsunobu conditions. Additionally, several further functional transformations of 4-methylenechromanes or their precursors were carried out to illustrate the synthetic potential of these intermediates.

\section{Introduction}

Multisubstituted chromane motifs are prevalent subunits in a series of bioactive natural products as well as in a variety of pharmaceutical agents. ${ }^{1}$ Some noteworthy examples are tocopherols and tocotrienols, ${ }^{2}$ which act as antioxidants to prevent lipid peroxidation and quench reactive oxygen species, nebivolol to treat hypertension, ${ }^{3}$ troglitazone as antidiabetic, ${ }^{4}$ repinotan as neuroprotector, ${ }^{5}$ (S)-equol as potent phytoestrogen $^{6}$ and ormeloxifene as anticancer agent. ${ }^{7}$<smiles></smiles>

$\alpha$-Tocopherol<smiles>COc1ccc(CC2SC(=O)NC2=O)cc1</smiles>

Nebivolol

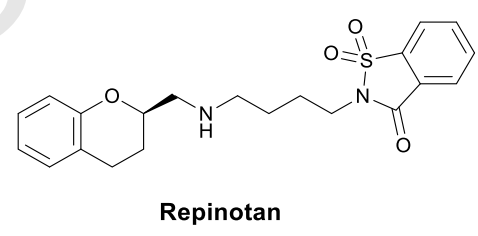

(S)-Equol

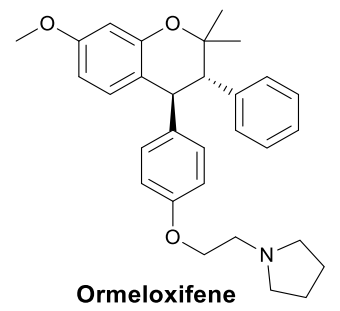

Figure 1 Some examples of bioactive compounds containing the chroman skeleton

Chromane have also been recognized as important intermediates in organic synthesis and the presence of functional groups on the benzopyran backbone can be a decisive advantage to access more complex and diverse

a Univ Rennes, CNRS, ISCR (Institut des Sciences Chimiques de Rennes) - UMR 6226, F-35000 Rennes, France. E-mail: bertrand.carboni@univ-rennes1.fr.

${ }^{b}$ Laboratoire de Synthèse des Molécules d'Intérêts Biologiques, Université des Frères Mentouri-Constantine, 25000 Constantine, Algérie.

Electronic Supplementary Information (ESI) available: Characterization and NMR spectra of all compounds, crystallographic data. See DOI: 10.1039/x0xx00000x structures. Given the multifaceted reactivity of the carboncarbon double bond, 4-alkylidenechromanes thus appear to be highly attractive building blocks to reach a wide range of new derivatives, ${ }^{8}$ targeted for their biological properties, as well as for their inherent potential for subsequent chemical transformations. This class of heterocycles can be synthesized from preformed chroman-4-ones by Wittig, Tebbe, Peterson, Mc Murry, Knoevenagel and Torgov reactions ${ }^{9}$ or via an organomagnesium addition/dehydration sequence. ${ }^{10}$ Alternately, the fused pyran ring can be constructed via transition metal-catalyzed reactions: intramolecular Heck 
coupling ${ }^{11}$ or $\mathrm{Rh}(\mathrm{III})$ - or $\mathrm{Pd}(\mathrm{II})$-catalyzed $\mathrm{C}-\mathrm{H}$ alkenylations, ${ }^{12}$ carbonickelation or palladation of the triple bond of iodoaryl propargylic compounds, ${ }^{13} \mathrm{Rh}(\mathrm{III})-$, $\mathrm{Au}(\mathrm{I})$-, Co (III) or $\operatorname{Ir}(\mathrm{I})$ catalyzed intramolecular hydroarylation. ${ }^{14} \mathrm{~A}$ metal-free approach using allylphenyl ethers as precursors and $I p y_{2} B_{4}$ is also reported. ${ }^{15}$ Miscellaneous other methods include enantioselective fluoration of alkenes, ${ }^{16}$ intramolecular trapping of allenylzincs, ${ }^{17}$ eliminative cross-coupling of $\mathrm{sp}^{3}$ hybridized carbenoids, ${ }^{18}$ silaborative carbocyclizations of $1,7-$ enynes, ${ }^{19}$ and nickel-catalyzed conjugative addition of $\mathrm{Me}_{2} \mathrm{Zn}$ to aldehydes across $1, \omega$-dienynes. ${ }^{20}$

Herein, we report a new access to 4-methylenechromanes 1 from 2-borylated $\alpha$-methylstyrenes (Scheme 1). This sequence is based on a key glyoxylate-ene reaction catalyzed by scandium (III) triflate, followed by a Mitsunobu cyclization after oxidation of the boronate function.

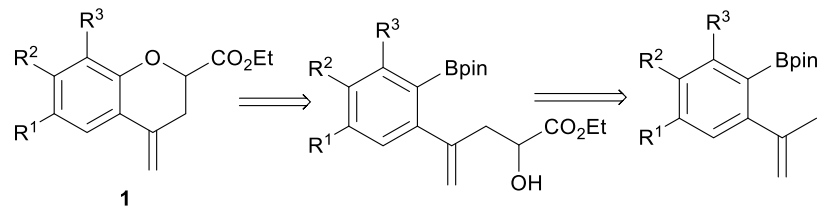

Scheme 1 Syntheses of 4-methylenechromanes from 2-borylated $\alpha$-methylstyrenes

\section{Results and discussion}

Initial studies began with the synthesis of the starting aryl boronates 4 according to two different approaches. Wittig reactions with methylenetriphenylphosphorane on commercially available or easily prepared 2bromoacetophenones $\mathbf{2}$ afforded the corresponding alkenes $\mathbf{3}$, which were converted in moderate to good yields to $\mathbf{4}$ by palladium-catalyzed borylation with bis(pinacolato)diboron in the presence of potassium acetate. During this step, variable amounts ( 5 to $10 \%$ ) of $\beta$, $\beta$-disubstituted vinyl boronates 5 were produced via an aryl to vinyl palladium 1,4-migration. ${ }^{21}$ Purification by chromatography on silica gel allowed the easy elimination of these undesired regioisomers (Table 1).

Table 1 Synthesis of boronates 4 via a Wittig / borylation sequence ${ }^{a}$
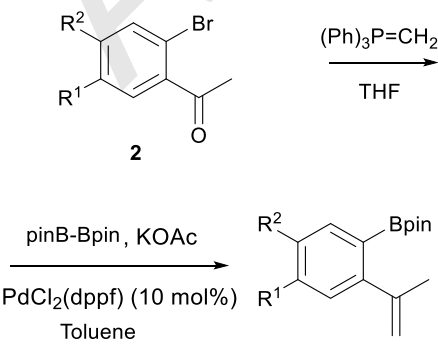

4
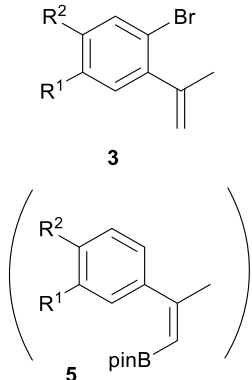

\begin{tabular}{lllll} 
Entry & $\mathrm{R}^{1}$ & $\mathrm{R}^{2}$ & $\mathbf{3}\left(\right.$ Yield \%) $^{\mathrm{b}}$ & $\mathbf{4}\left(\right.$ Yield \%) $^{\mathrm{b}}$ \\
\hline 1 & $\mathrm{H}$ & $\mathrm{H}$ & $\mathbf{3 a}(71)$ & $\mathbf{4 a}(73)$ \\
2 & $\mathrm{H}$ & $\mathrm{F}$ & $\mathbf{3 b}(62)$ & $\mathbf{4 b}(48)$ \\
3 & $\mathrm{H}$ & OMe & $\mathbf{3 c}(56)$ & $\mathbf{4 c}(69)$ \\
4 & $\mathrm{Me}$ & OMe & $\mathbf{3 d}(43)$ & $\mathbf{4 d}(64)$ \\
5 & $\mathrm{NO}_{2}$ & $\mathrm{H}$ & $\mathbf{3 e}(49)$ & $\mathbf{4 e}(41)$ \\
6 & $\mathrm{Cl}$ & $\mathrm{H}$ & $\mathbf{3 f}(61)^{\mathrm{c}}$ & $\mathbf{4 f}(62)$
\end{tabular}

a Reactions conditions: (a) $\mathrm{Ph}_{3} \mathrm{PCH}_{3} \mathrm{Br}$ (1.05 equiv.), $\mathrm{n}$-BuLi (1 equiv.), $\mathrm{THF}, 0^{\circ} \mathrm{C}$ to $\mathrm{rt}$, 16h; (b) PinBBPin (2.5 equiv.), KOAc (2.5 equiv.), $\mathrm{PdCl}_{2}$ (dppf) $\left(5 \mathrm{~mol} \%\right.$ ), toluene, $80^{\circ} \mathrm{C}$, 15h. ${ }^{\text {b }}$ Yields of isolated products after purification by chromatography. ${ }^{\mathrm{C}}$ Unlike other 2 bromo $\alpha$-methylstyrenes, $\mathbf{3 f}$ was prepared according to the following sequence: addition of methylmagnesium bromide to methyl 2-bromo-5-chlorobenzoate and dehydration. ${ }^{22}$ The yield is given for the combined two steps.

The second route involves speciation control during the Suzuki-Miyaura coupling of pinacol isopropenylboronate with 2-bromoaryl MIDA boronic esters, ${ }^{23}$ which were easily synthesized from the corresponding boronic acids for $\mathbf{6 a}, \mathbf{g}-\mathbf{h} 24$ and by bromination of the corresponding acetanilide for $6 \mathbf{i}$ (Table 2). ${ }^{25}$

\section{Table 2 Synthesis of boronates 4 from B-MIDA derivatives ${ }^{\text {a }}$}

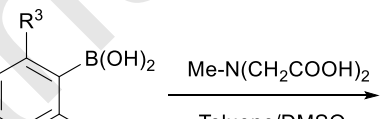

$$
\begin{aligned}
& \text { Toluene/DMSO }
\end{aligned}
$$<smiles>[R]c1cc([R3])c(Br)c(Br)c1</smiles>

$6 a, g, h$

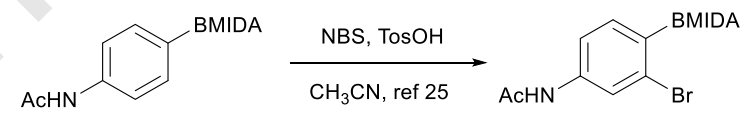

$6 \mathrm{i}$

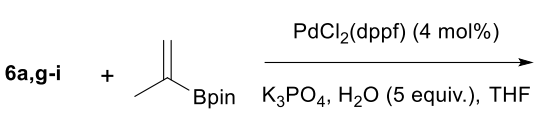

\begin{tabular}{lllll}
\hline Entry & $\mathrm{R}^{1}$ & $\mathrm{R}^{3}$ & $\mathbf{6}\left(\right.$ Yield \%) $^{\mathrm{b}}$ & $\mathbf{4}\left(\right.$ Yield \%) $^{\mathrm{b}}$ \\
\hline 1 & $\mathrm{H}$ & $\mathrm{H}$ & $\mathbf{6 a}(88)$ & $\mathbf{4 a}(74)$ \\
2 & $\mathrm{H}$ & $\mathrm{F}$ & $\mathbf{6 g}(86)$ & $\mathbf{4 g}(35)$ \\
3 & $\mathrm{Me}$ & $\mathrm{H}$ & $\mathbf{6 h}(87)$ & $\mathbf{4 h}(50)$ \\
4 & AcNH & $\mathrm{H}$ & $\mathbf{6 i}(62)^{\mathrm{c}}$ & $\mathbf{4 i}(54)$
\end{tabular}

a Reactions conditions: (a) $\mathrm{N}$-methyliminodiacetic acid (1 equiv.), toluene/DMSO (5/1), reflux, 20 h. (b) NBS, $p-\mathrm{MeC}_{6} \mathrm{H}_{4} \mathrm{SO}_{3} \mathrm{H}, \mathrm{MeCN}, \mathrm{rt}, 24$ h. (c) 4,4,5,5-tetramethyl-2(prop-1-en-2-yl)-1,3,2-dioxaborolane (1.5 equiv.), $\mathrm{PdCl}_{2}$ (dppf) (4 mol\%), $\mathrm{K}_{3} \mathrm{PO}_{4}$ (3 equiv.), $\mathrm{H}_{2} \mathrm{O}$ (5 equiv.), THF, $90^{\circ} \mathrm{C}, 20 \mathrm{~h}$ for $4 \mathrm{a}, \mathrm{g}-\mathrm{h}$ and $40^{\circ} \mathrm{C}, 20 \mathrm{~h}$ for $4 \mathrm{i}^{\mathrm{b}}{ }^{\mathrm{b}}$ Yields of isolated products after purification by chromatography or Kugelrohr distillation. ${ }^{\mathrm{c}}$ The yield is given for the combined two steps (96x65\%).

After optimization of the reaction conditions, the best conversions to the desired boronates were observed at $90{ }^{\circ} \mathrm{C}$ for $20 \mathrm{~h}$, except for $4 \mathbf{i}\left(40^{\circ} \mathrm{C}\right)$ due its low thermal stability. Purifications were usually carried out by column chromatography on silica gel. $\mathbf{4 g}$ required additional Kugelrohr 
distillation, which may explain the lower final yield. ${ }^{1} \mathrm{H},{ }^{13} \mathrm{C},{ }^{11} \mathrm{~B}$ NMR data are in full agreement with the proposed structures.

Ene reactions have been widely employed in organic synthesis to create a new carbon-carbon bond in the allylic position of an alkene. ${ }^{26,27}$ While a large variety of functionalized reactants have been used, there are still scarce examples of boron derivatives. ${ }^{28}$ As part of our effort intended at demonstrating the synthetic utility of unsaturated boronates in such processes, ${ }^{28 a}$ we then engaged the previously prepared 2-borylated $\alpha$-methylstyrenes 4 in carbonyl-ene reaction with ethyl glyoxylate. In the presence of scandium (III) triflate in methylene chloride, they were actually converted into the expected $\alpha$-hydroxy esters 7 (Table 3 ). If aryl substituents, such as $\mathrm{F}, \mathrm{Cl}, \mathrm{OMe}$, Me, were well tolerated, despite several attempts, only traces of ene-product were detected from the nitro derivative $4 \mathbf{e} .^{29}$ Other catalytic systems as $\ln (\mathrm{OTf})_{3}, \mathrm{Yb}(\mathrm{OTf})_{3}, \mathrm{La}(\mathrm{OTf})_{3}$ did not bring any noticeable improvements. 7 showed a low stability on silica gel during the purification process, mainly due to its conversion to 1,3,4,5-tetrahydrobenzo[c][1,2]oxaborepines $\mathbf{8}$, the sevenmembered ring analogue of benzoxaborole. ${ }^{30}$ This preference for the closed form has been thoroughly studied for the open corresponding boronic acid. ${ }^{30 a}$ In our case, the cyclization of the pinacol ester is probably due to the acidic nature of the silica gel and the same observation was made with $2 \mathrm{~N}$ aqueous $\mathrm{HCl}$ in acetone. Unfortunately, if we succeeded in isolating several pure compounds $\mathbf{8}$ with variable yields, this conversion was never complete, which limits, at the moment, the use of this approach as an efficient access to these uncommon boron bicyclic derivatives. The cyclic and open forms 7 and 8 are clearly differentiated via their ${ }^{1} \mathrm{H}$ NMR spectra. For example, concerning the parent derivative, it was noted the absence of pinacol signals for 8a (see supporting information), welldefined double doublet at $2.87 \mathrm{ppm}(J=8.8$ and $13.6 \mathrm{~Hz})$ and double double doublet at $3.10 \mathrm{ppm}(J=1.3,3.3$ and $13.6 \mathrm{~Hz})$ for the two allylic hydrogens of 7a while a complex nonanalyzable multiplet at 3.11-3.06 ppm was observed for 8a. A significant chemical shift difference of $0.6 \mathrm{ppm}$ was also measured for the hydrogen $\alpha$ to the ester group (4.19 ppm for $7 \mathrm{a}$ and $4.82 \mathrm{ppm}$ for $8 \mathrm{a}) .{ }^{13} \mathrm{C},{ }^{11} \mathrm{~B}$ NMR and HRMS confirm the proposed structures and are in agreement with the literature. ${ }^{30}$

In order to overcome significant losses during the purification of 7 , the crude ene-product was directly engaged in the subsequent oxidation reaction using sodium perborate during one night at room temperature. ${ }^{31}$ The phenols 9 were isolated by purification on silica gel with moderate overall yields ( 2 steps) (Table 3 ). It is noteworthy that, in the case of 9c and 9d which have a methoxy electron-donating group on the aryl part, a prolonged stay on the column or simply in $\mathrm{CDCl}_{3}$ in the NMR tube causes a partial isomerization of the double bond to give the corresponding trisubstituted alkenes. Cyclisation to 4-methylenechromanes 1 was then performed in good yields using standard Mitsunobu conditions with diisopropyl azodicarboxylate and triphenylphosphine. As previously, for $\mathbf{1 c}$ and $\mathbf{1 d}$, the presence of the methoxy substituent facilitates the migration of the double bond in the endocyclic position during the purification step. Complete conversion to $10 \mathrm{c}$ and $10 \mathrm{~d}$ was observed after treatment with trifluoroacetic acid (see below).

Table 3. Ene reaction/ oxidation of boronates $4^{\text {a }}$
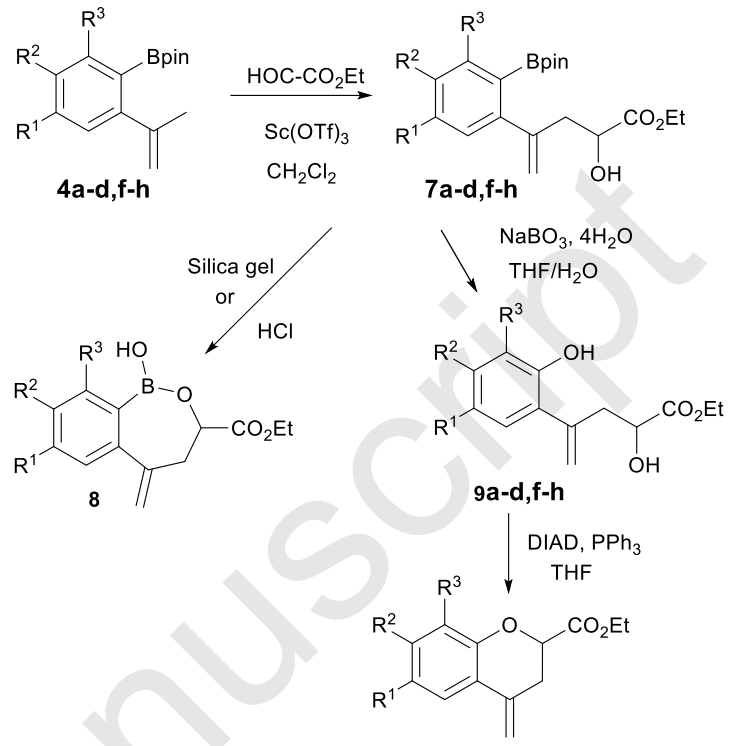

1a-d,f-h

\begin{tabular}{llllll}
\hline Entry & $\mathrm{R}^{1}$ & $\mathrm{R}^{2}$ & $\mathrm{R}^{3}$ & 9 (Yield \%), & 1 (Yield \%) \\
\hline 1 & $\mathrm{H}$ & $\mathrm{H}$ & $\mathrm{H}$ & $\mathbf{9 a}(58)$ & $\mathbf{1 a ~ ( 7 9 )}$ \\
$\mathbf{2}$ & $\mathrm{H}$ & $\mathrm{F}$ & $\mathrm{H}$ & $\mathbf{9 b}(34)$ & $\mathbf{1 b}(66)$ \\
3 & $\mathrm{H}$ & OMe & $\mathrm{H}$ & $\mathbf{9 c}(52)$ & $\mathbf{1 c}(80)^{\mathrm{d}}$ \\
4 & $\mathrm{Me}$ & $\mathrm{OMe}$ & $\mathrm{H}$ & $\mathbf{9 d}(54)$ & $\mathbf{1 d ( 5 8 )}$ \\
5 & $\mathrm{Cl}$ & $\mathrm{H}$ & $\mathrm{H}$ & $\mathbf{9 f}(49)$ & $\mathbf{1 f}(76)$ \\
6 & $\mathrm{H}$ & $\mathrm{H}$ & $\mathrm{F}$ & $\mathbf{9 g}(41)$ & $\mathbf{1 g}(88)$ \\
7 & $\mathrm{Me}$ & $\mathrm{H}$ & $\mathrm{H}$ & $\mathbf{9 h}(54)$ & $\mathbf{1 h}(77)$
\end{tabular}

a Reactions conditions: (a) pre-dried scandium triflate $(10 \mathrm{~mol} \%)$, ethyl glyoxylate $(1.5$ equiv., $50 \%$ in toluene), $\mathrm{DCM}$, rt, overnight. (b) crude boronate 7 , sodium perborate tetrahydrate (3 equiv.), THF/ $\mathrm{H}_{2} \mathrm{O}(2 / 1)$, rt, overnight. (c) triphenylphosphine (1 equiv.), diisopropyl azodicarboxylate (1 equiv.), DCM, rt, overnight. ${ }^{b}$ Yields of isolated products after purification by chromatography on silica gel. ${ }^{\mathrm{c}}$ The yield is given for the combined two steps, ene reaction/oxidation. ${ }^{d}$ Mixture of exo-/endocyclic isomers: 53/47. e Mixture of exo-/endocyclic isomers: 90/10.

Finally, we briefly explored some functional transformations of 4-methylenechromanes 1 and boronate 7a to illustrate the synthetic potential of these compounds (Scheme 2). Isomerisation of $\mathbf{1}$ to chromenes $\mathbf{1 0}$ was quantitatively achieved under trifluoroacetic acid catalysis. ${ }^{32}$ Hydrogenation of $1 \mathbf{a}$ in the presence of Pd on charcoal under atmospheric hydrogen gas pressure afforded the chromane 11a as a mixture of two diastereomers $(87 / 13)$. 


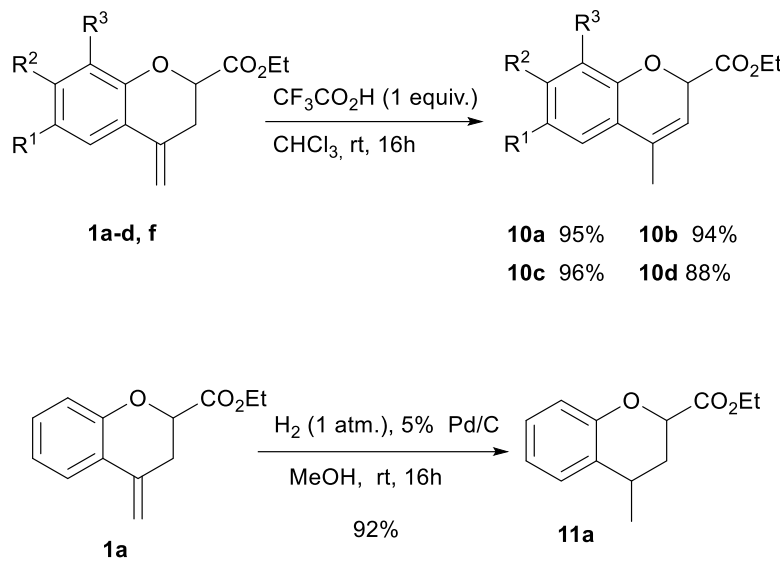

Scheme 2 Functional transformations of 4-methylenechromanes 1

Concerning the boronate $\mathbf{7 a}$, it was first converted to the corresponding azide 12a via a copper-catalyzed azidation. ${ }^{33}$ Treatment with triphenylphosphine in THF afforded the iminophosphorane 13a which has been characterised by ${ }^{1} \mathrm{H}$, ${ }^{13} \mathrm{C},{ }^{31} \mathrm{P}$ NMR and HRMS. Its structure was unambiguously confirmed by single crystal X-ray analysis. ${ }^{34}$ The $\mathrm{N}(21)-\mathrm{H}-\mathrm{O}(32)$ angle is $147.8^{\circ}$ and the critical $\mathrm{N}(21)-\mathrm{O}(32)$ distance is $2.84 \AA$, within the accepted range for intramolecular hydrogen bonding, thus resulting in the formation of an unusual eightmembered ring in a boat-like conformation (Scheme 3, Figure 2). Hydrolysis of 13 a was performed in $\mathrm{THF} /$ water at $70^{\circ} \mathrm{C}$ for $16 \mathrm{~h}$ to the corresponding aniline $14 \mathrm{a}$ in $84 \%$ yield. Following the work of Kazmaier et al, ${ }^{35}$ we also prepared the indole $\mathbf{1 5 a}$ via a photochemical nitrene generation/ $\mathrm{C}-\mathrm{H}$ insertion sequence.

\section{Conclusion}

In summary, we have demonstrated a convenient access to 4methylenechromanes from 2-borylated $\alpha$-methylstyrenes via a scandium(III) triflate-catalyzed glyoxylate-ene reaction as key step. Some aspects of the synthetic utility of these important heterocyclic subunits as well as of their boronate precursors have been also explored. Further studies on the use of this approach in natural product synthesis, as heliannuol $E,{ }^{36}$ and on the optimization of the synthesis of 1.2-oxaborepanes are in progress in our laboratory.

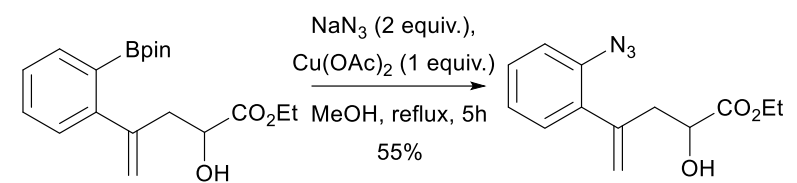

$7 a$<smiles></smiles>

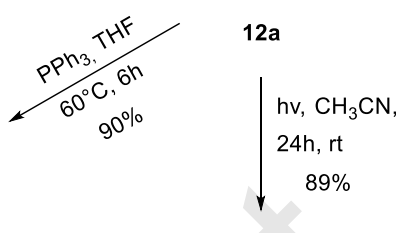<smiles>C=C(CC(O)C(=O)OCC)c1ccccc1N</smiles>

$14 \mathrm{a}$

Scheme 3 Functional transformations of isolated boronate 7a

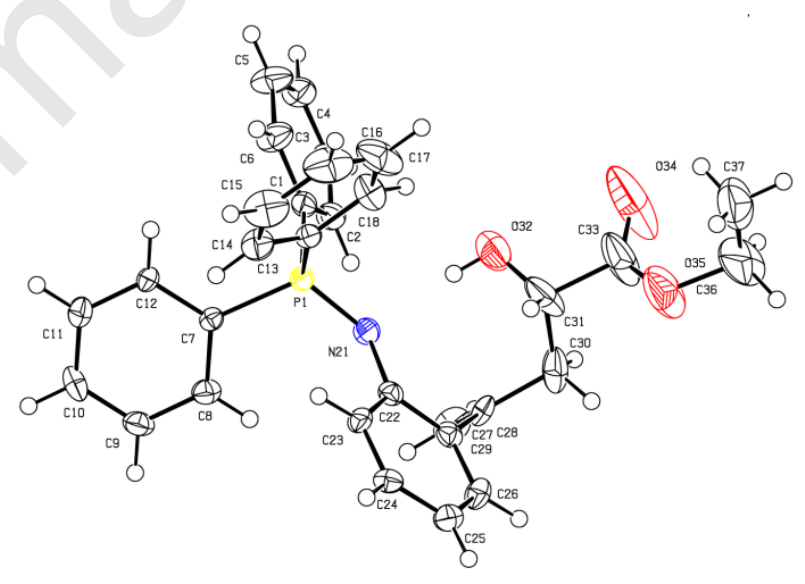

Figure 2 X-Ray crystallographic structure of iminophosphorane 13a

\section{Experimental section}

\section{General information}

Tetrahydrofuran (THF) and toluene (DCM) were distilled over sodium/benzophenone and dichloromethane over $\mathrm{P}_{2} \mathrm{O}_{5}$. NMR spectra were recorded on Bruker apparatus at 300 or $400 \mathrm{MHz}$ for ${ }^{1} \mathrm{H}, 75$ or $101 \mathrm{MHz}$ for ${ }^{13} \mathrm{C}, 282$ or $376 \mathrm{MHz}$ for ${ }^{19} \mathrm{~F}, 121 \mathrm{~Hz}$ for ${ }^{31} \mathrm{P}$ and 96 or $128 \mathrm{MHz}$ for ${ }^{11} \mathrm{~B}$. Chemical shifts of ${ }^{1} \mathrm{H}$ and ${ }^{13} \mathrm{C}$ NMR were referenced to $\mathrm{Me}_{4} \mathrm{Si}$ as internal reference. Data are represented as follows: chemical shift ( $\mathrm{ppm}$ ), multiplicity ( $\mathrm{s}=$ singlet, $\mathrm{d}=$ doublet, $\mathrm{t}=$ triplet, $\mathrm{q}=$ quartet, $\mathrm{m}=$ multiplet, $\mathrm{br}=$ broad), coupling constant $\mathrm{J}(\mathrm{Hz})$ and integration. All high- 
resolution mass spectra (HRMS) were recorded on a Bruker Micro-Tof-Q II or on a Waters Q-Tof 2 at the CRMPO (Centre Régional de Mesures Physiques de l'Ouest, Rennes, France) using positive ion electrospray. Purifications on silica gel were carried out on silica gel $0.006-0.200 \mathrm{~mm}, 60 \mathrm{~A}$. Analytical thin layer chromatography was performed on Merck Silica gel 60 $\mathrm{F}_{254}$ plates. Flash chromatography purifications were performed on a Grace Reveleris ${ }^{\mathrm{TM}}$ with Puriflash ${ }^{\mathrm{TM}} 15 \mu \mathrm{m}$ flash cartridges. X-ray crystallographic data were collected on an APEXII crystal diffractometer (Centre de Diffractométrie $X$, Institut des Sciences Chimiques de Rennes). Melting points were measured on a melting point apparatus Stuart SMP10.

\section{General procedure for the synthesis of styrenes (3) via a Wittig} reaction

A dry flask was charged under argon with a solution of methyltriphenylphosphonium bromide $(1.79 \mathrm{~g}, 5 \mathrm{mmol})$ in anhydrous THF $(20 \mathrm{~mL})$. $n$-BuLi $(3 \mathrm{~mL}, 1.6 \mathrm{M}$ in hexane, 4.8 $\mathrm{mmol}$ ) was added dropwise at $0^{\circ} \mathrm{C}$. After $60 \mathrm{~min}$, a solution of $o$-bromoacetophenone $(4.8 \mathrm{mmol})$ in $5 \mathrm{~mL}$ of THF was added dropwise at the same temperature. The reaction was then allowed to stir overnight at room temperature. The reaction mixture was quenched with saturated aqueous $\mathrm{NH}_{4} \mathrm{Cl}$ solution, extracted with $\mathrm{AcOEt}$, dried over $\mathrm{MgSO}_{4}$ and concentrated under vacuum. Addition of pentane caused the precipitation of triphenylphosphine oxide which was discarded by filtration. The filtrate was concentred under vacuum. Purification with distillation or chromatography on silica gel afforded the corresponding 2-bromostyrene derivatives 3.

\section{1-Bromo-2-(prop-1-en-2-yl)benzene (3a)}

Colorless oil, $671 \mathrm{mg}, 71 \%$; bp $40-45{ }^{\circ} \mathrm{C}$ at $0.1 \mathrm{mmHg}$. ${ }^{1} \mathrm{H}$ NMR (300 MHz, Chloroform- $d, \delta$ ppm) 7.55 (dd, $J=8.0,1.2 \mathrm{~Hz}, 1 \mathrm{H}$ ), 7.26 (ddd, $J=7.6,7.1,1.2 \mathrm{~Hz}, 1 \mathrm{H}$ ), 7.20 (dd, $J=7.6,2.0 \mathrm{~Hz}, 1 \mathrm{H}$ ), $7.11(\mathrm{ddd}, J=8.0,7.1,2.0 \mathrm{~Hz}, 1 \mathrm{H}), 5.23(\mathrm{dq}, J=1.9,1.6 \mathrm{~Hz}, 1 \mathrm{H})$, 4.95 (dq, $J=1.9,0.9 \mathrm{~Hz}, 1 \mathrm{H}), 2.11(\mathrm{dd}, J=1.6,0.9 \mathrm{~Hz}, 3 \mathrm{H}) .{ }^{13} \mathrm{C}$ NMR (101 MHz, Chloroform-d, $\delta$ ppm) 145.9, 145.0, 132.9, $129.9,128.5,127.4,121.7,116.1,23.7$. The spectroscopic data match with those reported in the literature. ${ }^{37}$

\section{2-Bromo-4-fluoro-1-(prop-1-en-2-yl)benzene (3b)}

Colorless oil, $640 \mathrm{mg}, 62 \%$; b.p. $45-48{ }^{\circ} \mathrm{C}$ at $0.1 \mathrm{mmHg} .{ }^{1} \mathrm{H}$ NMR (400 MHz, Chloroform- $d, \delta$ ppm) 7.32 (dd, $J=8.4,2.6 \mathrm{~Hz}, 1 \mathrm{H}$ ), $7.18(\mathrm{dd}, J=8.5,6.1 \mathrm{~Hz}, 1 \mathrm{H}), 7.00$ (ddd, $J=8.5,8.4,2.6 \mathrm{~Hz}, 1 \mathrm{H}$ ), 5.25 (quint, $J=1.5 \mathrm{~Hz} 1 \mathrm{H}$ ), $4.95(\mathrm{q}, J=0.9 \mathrm{~Hz}, 1 \mathrm{H}), 2.10(\mathrm{t}, J=$ $1.1 \mathrm{~Hz}, 3 \mathrm{H}) .{ }^{13} \mathrm{C}$ NMR (75 MHz, Chloroform-d, $\left.\delta \mathrm{ppm}\right) 161.3$ (d, J $=249.5 \mathrm{~Hz}$ ), 144.9, $140.9(\mathrm{~d}, J=3.6 \mathrm{~Hz}), 130.5(\mathrm{~d}, J=8.2 \mathrm{~Hz})$, $121.6(\mathrm{~d}, J=9.5 \mathrm{~Hz}), 119.8(\mathrm{~d}, J=24.4 \mathrm{~Hz}), 116.6,114.3(\mathrm{~d}, J=$ 20.8 Hz), 23.6. ${ }^{19} \mathrm{~F} \mathrm{NMR} \mathrm{(376} \mathrm{MHz,} \mathrm{Chloroform-d,} \delta \mathrm{ppm}$ ) 114.1. The spectroscopic data match with those reported in the literature. ${ }^{38}$

\section{2-Bromo-4-methoxy-1-(prop-1-en-2-yl)benzene (3c)}

Colorless oil, $610 \mathrm{mg}, 56 \%$; bp $50-55{ }^{\circ} \mathrm{C}$ at $0.1 \mathrm{mmHg} .{ }^{1} \mathrm{H}$ NMR (300 MHz, Chloroform- $d, \delta$ ppm) 7.11 (d, $J=2.6 \mathrm{~Hz}, 1 \mathrm{H}$ ), 7.10 $(\mathrm{d}, J=8.4 \mathrm{~Hz}, 1 \mathrm{H}), 6.81(\mathrm{dd}, J=8.4,2.6 \mathrm{~Hz}, 1 \mathrm{H}), 5.20$ (dq, $J=$ 1.9, $1.5 \mathrm{~Hz}, 1 \mathrm{H}), 4.92(\mathrm{dq}, J=1.9,0.9 \mathrm{~Hz}, 1 \mathrm{H}), 3.79(\mathrm{~s}, 3 \mathrm{H}), 2.07$ (dd, $J=1.5,0.9 \mathrm{~Hz}, 3 \mathrm{H}) .{ }^{13} \mathrm{C}$ NMR $(75 \mathrm{MHz}$, Chloroform-d, $\delta$ ppm) 159.0, 145.5, 137.3, 130.3, 121.8, 117.9, 116.2, 113.5, $55.7,23.9$. The spectroscopic data match with those reported in the literature. ${ }^{39}$

\section{1-Bromo-5-methoxy-4-methyl-2-(prop-1-en-2-yl)benzene (3d)}

Colorless oil, $497 \mathrm{mg}, 43 \%$; bp $50-55{ }^{\circ} \mathrm{C}$ at $0.1 \mathrm{mmHg} .{ }^{1} \mathrm{H}$ NMR (300 MHz, Chloroform- $d, \delta$ ppm) 6.99-6.94 (m, 2H), 5.18 (dq, J $=1.9,1.6 \mathrm{~Hz}, 1 \mathrm{H}), 4.90(\mathrm{dq}, J=1.9,0.9 \mathrm{~Hz}, 1 \mathrm{H}), 3.81(\mathrm{~s}, 3 \mathrm{H})$, $2.15(\mathrm{~s}, 3 \mathrm{H}), 2.07$ (dd, $J=1.6,0.9 \mathrm{~Hz}, 3 \mathrm{H}) .{ }^{13} \mathrm{C}$ NMR $(101 \mathrm{MHz}$, Chloroform- $d, \delta$ ppm) 157.1, 145.6, 136.6, 131.4, 125.9, 118.4, $115.9,114.4,55.7,23.9,15.8$. HRMS $\left(\mathrm{ESI}^{+}\right)$calcd. for $[\mathrm{M}+\mathrm{H}]^{+}$ $\left(\mathrm{C}_{11} \mathrm{H}_{14} \mathrm{O}^{79} \mathrm{Br}\right)$ : 241.0222, found 241.0225 .

\section{1-Bromo-4-nitro-2-(prop-1-en-2-yl)benzene (3e)}

Colorless oil, $569 \mathrm{mg}, 49 \% .{ }^{1} \mathrm{H}$ NMR (300 MHz, Chloroform- $d, \delta$ ppm) 8.05 (d, $J=2.8 \mathrm{~Hz}, 1 \mathrm{H}), 7.97$ (dd, $J=8.7,2.7 \mathrm{~Hz}, 1 \mathrm{H}), 7.73$ (d, $J=8.7 \mathrm{~Hz}, 1 \mathrm{H}$ ), 5.34 (quint, $J=1.5 \mathrm{~Hz}, 1 \mathrm{H}$ ), $5.03(\mathrm{q}, J=1.1$ $\mathrm{Hz}, 1 \mathrm{H}), 2.16-2.07$ (m, 3H). ${ }^{13} \mathrm{C}$ NMR (101 MHz, Chloroform-d, $\delta$ ppm) 147.1, 146.3, 143.9, 133.9, 129.3, 124.5, 123.0, 117.9, 23.1. HRMS $\left(E S I^{+}\right)$calcd. for $[\mathrm{M}+\mathrm{Na}]^{+}\left(\mathrm{C}_{9} \mathrm{H}_{8} \mathrm{NO}_{2}{ }^{79} \mathrm{BrNa}\right)$ : 263.9630, found: 263.9634 .

\section{Synthesis of 1-bromo-4-chloro-2-(prop-1-en-2-yl)benzene (3f)}

A solution of methyl 2-bromo-5-chlorobenzoate $(2.45 \mathrm{~g}, 10$ $\mathrm{mmol})$ in $\mathrm{Et}_{2} \mathrm{O}(20 \mathrm{~mL})$ was placed in a $100 \mathrm{~mL}$ Schlenk tube under argon. To this solution cooled to $0{ }^{\circ} \mathrm{C}$ was added dropwise $\mathrm{MeMgBr}\left(3 \mathrm{M}\right.$ in $\mathrm{Et}_{2} \mathrm{O}, 10 \mathrm{~mL}, 30.0 \mathrm{mmol}$ ) over 30 $\mathrm{min}$. The solution was stirred for $48 \mathrm{~h}$ at room temperature. After slowly quenching with a saturated aqueous solution of $\mathrm{NH}_{4} \mathrm{Cl}(10 \mathrm{~mL})$, followed by $\mathrm{HCl}(2 \mathrm{M}, 10 \mathrm{~mL})$, the aqueous layer was extracted with EtOAc $(3 \times 20 \mathrm{~mL})$. The combined organic layers were washed with brine $(10 \mathrm{~mL})$, dried with $\mathrm{Mg}_{2} \mathrm{SO}_{4}$, and concentrated in vacuo to afford the crude alcohol as oil which was used without further purification.

To a solution of the above alcohol in chloroform (15 $\mathrm{mL}$ ) was added concentrated $\mathrm{H}_{2} \mathrm{SO}_{4}(150 \mu \mathrm{L})$. The solution was refluxed for $4.5 \mathrm{~h}$ and then quenched with a saturated aqueous solution of $\mathrm{NaHCO}_{3}(25 \mathrm{~mL})$. The aqueous layer was extracted with EtOAc $(3 \times 15 \mathrm{~mL})$. The combined organic layers were washed with brine $(20 \mathrm{~mL})$, dried with $\mathrm{MgSO}_{4}$, and concentrated in vacuo to afford the crude olefin. Purification by column chromatography on silica gel (cyclohexane/EtOAc=95/5) afforded $\mathbf{3 f}$ as a colorless oil ( $1.41 \mathrm{~g}, 61 \%$ over two steps).

\section{1-Bromo-4-chloro-2-(prop-1-en-2-yl)benzene (3f)}

Colorless oil, $1.41 \mathrm{~g}, 61 \%$; ${ }^{1} \mathrm{H}$ NMR (300 MHz, Chloroform- $d, \delta$ ppm) $7.48(\mathrm{~d}, J=8.5 \mathrm{~Hz}, 1 \mathrm{H}), 7.20(\mathrm{~d}, J=2.6 \mathrm{~Hz}, 1 \mathrm{H}), 7.11$ (dd, $J$ $=8.5,2.6 \mathrm{~Hz}, 1 \mathrm{H}), 5.28-5.25(\mathrm{~m}, 1 \mathrm{H}), 4.99-4.96(\mathrm{~m}, 1 \mathrm{H}), 2.11-$ $2.08(\mathrm{~m}, 3 \mathrm{H}) .{ }^{13} \mathrm{C}$ NMR (75 MHz, Chloroform-d, $\delta \mathrm{ppm}$ ) 146.3, 144.7, 133.8, 133.1, 129.6, 128.4, 119.5, 116.8, 23.2. HRMS $\left(\mathrm{ESI}^{+}\right)$calcd. for [M] ${ }^{+\cdot}\left(\mathrm{C}_{9} \mathrm{H}_{8}{ }^{35} \mathrm{Cl}^{79} \mathrm{Br}\right)$ : 229.9492 , found: 229.9496 . 
General procedure for the synthesis of boronates (4a-f) by borylation of (3a-f)

To a solution of 2-bromo-1-(prop-1-en-2-yl) benzene 3 (1 $\mathrm{mmol}$ ) in dry toluene $(5 \mathrm{~mL}$ ) was added bis (pinacolato)diboron (635 $\mathrm{mg}, 2.5 \mathrm{mmol}$ ) and anhydrous potassium acetate (245 $\mathrm{mg}, 2.5 \mathrm{mmol}$ ). The reaction system was degassed with argon, before the addition of $\mathrm{PdCl}_{2}$ (dppf) (36 mg, $5 \mathrm{~mol} \%$ ). The solution was stirred at $80{ }^{\circ} \mathrm{C}$ overnight, cooled to $\mathrm{rt}$, diluted with AcOEt and filtered. The filtrate was evaporated in vacuo and the residue was purified by column chromatography (cyclohexane/AcOEt : 10/3).

\section{4,4,5,5-Tetramethyl-2-(2-(prop-1-en-2-yl)phenyl)-1,3,2- dioxaborolane (4a)}

Colorless oil, $178 \mathrm{mg}, 73 \%$; ${ }^{1} \mathrm{H}$ NMR (300 MHz, Chloroform- $d$, $\delta$ ppm) 7.65-7.60 (m, 1H), 7.38-7.32 (m, 1H), 7.26-7.20 (m, 2H), 5.06 (quint, $J=1.5 \mathrm{~Hz}, 1 \mathrm{H}$ ), $4.88(\mathrm{dq}, J=1.9,0.9 \mathrm{~Hz}, 1 \mathrm{H}), 2.14$ (dd, $J=1.6,0.9 \mathrm{~Hz}, 3 \mathrm{H}), 1.33(\mathrm{~s}, 12 \mathrm{H}) .{ }^{13} \mathrm{C}$ NMR $(101 \mathrm{MHz}$, Chloroform- $d, \delta$ ppm) 149.6, 147.6, 134.3, 130.0, 127.0, 126.1, $114.2,83.6,24.7,24.5$ (the carbon $\alpha$ to boron was not found). ${ }^{11} \mathrm{~B}$ NMR (128 MHz, Chloroform-d, $\left.\delta \mathrm{ppm}\right)$ 31.0. HRMS (ESI+) found for $[\mathrm{M}+\mathrm{Na}]^{+}\left(\mathrm{C}_{15} \mathrm{H}_{21} \mathrm{O}_{2}{ }^{11} \mathrm{BNa}\right)$ : 267.1526, found: 267.1528

\section{2-(5-Fluoro-2-(prop-1-en-2-yl)phenyl)-4,4,5,5-tetramethyl- 1,3,2-dioxaborolane (4b)}

Colorless oil, $126 \mathrm{mg}, 48 \%$; Yield $=48 \% .{ }^{1} \mathrm{H}$ NMR $(300 \mathrm{MHz}$ Chloroform- $d, \delta$ ppm) 7.29 (dd, $J=8.5,2.8 \mathrm{~Hz}, 1 \mathrm{H}$ ), 7.18 (dd, $J$ $=8.5,5.3 \mathrm{~Hz}, 1 \mathrm{H}), 7.02(\mathrm{dd}, J=8.5,8.5,2.8 \mathrm{~Hz}, 1 \mathrm{H}), 5.06(\mathrm{dq}, J$ $=1.9,1.6 \mathrm{~Hz}, 1 \mathrm{H}), 4.85(\mathrm{dq}, J=1.9,0.9 \mathrm{~Hz}, 1 \mathrm{H}), 2.11(\mathrm{dd}, J=$ 1.6, $0.9 \mathrm{~Hz}, 3 \mathrm{H}), 1.32$ (s, 12H). ${ }^{13} \mathrm{C}$ NMR $(101 \mathrm{MHz}$, Chloroformd, $\delta \mathrm{ppm}) 161.3(\mathrm{~d}, J=246.0 \mathrm{~Hz}), 146.5,145.4(\mathrm{~d}, J=3.1 \mathrm{~Hz})$, $128.8(\mathrm{~d}, J=7.3 \mathrm{~Hz}), 120.4(\mathrm{~d}, J=19.6 \mathrm{~Hz}), 116.5(\mathrm{~d}, J=21.1$ $\mathrm{Hz}$ ), 114.5, 83.9, 24.7, 24.6 (the carbon $\alpha$ to boron was not found). ${ }^{19} \mathrm{~F}$ NMR (376 MHz, Chloroform-d, $\delta$ ppm) -117.4. ${ }^{11} \mathrm{~B}$ NMR (128 MHz, Chloroform-d, $\delta$ ppm) 31.2. HRMS (ESI ${ }^{+}$) calcd. for $[\mathrm{M}+\mathrm{Na}]^{+}\left(\mathrm{C}_{15} \mathrm{H}_{20} \mathrm{O}_{2} \mathrm{~F}^{11} \mathrm{BNa}\right)$ : 285.1432, found: 285.1435 .

2-(5-Methoxy-2-(prop-1-en-2-yl)phenyl)-4,4,5,5-tetramethyl1,3,2-dioxaborolane (4c)

Colorless oil, $189 \mathrm{mg}, 69 \%$; ${ }^{1} \mathrm{H}$ NMR (300 MHz, Chloroform- $d$, $\delta$ ppm) 7.17 (dd, $J=8.5,0.4 \mathrm{~Hz}, 1 \mathrm{H}$ ), 7.13 (dd, $J=2.9,0.4 \mathrm{~Hz}, 1 \mathrm{H}$ ), $6.89(\mathrm{dd}, J=8.5,2.9 \mathrm{~Hz}, 1 \mathrm{H}), 5.04-5.00(\mathrm{~m}, 1 \mathrm{H}), 4.85(\mathrm{dq}, J=$ 1.9, $0.9 \mathrm{~Hz}, 1 \mathrm{H}$ ), $3.81(\mathrm{~s}, 3 \mathrm{H}), 2.11$ (dd, $J=1.5,0.9 \mathrm{~Hz}, 3 \mathrm{H}), 1.32$ (s, $12 \mathrm{H}) .{ }^{13} \mathrm{C}$ NMR $(75 \mathrm{MHz}$, Chloroform-d, $\delta \mathrm{ppm}) 157.8,146.9$, $142.0,128.3,118.8,115.6,113.82,83.7,55.3,26.9,24.6$ (the carbon $\alpha$ to boron was not found). ${ }^{11} \mathrm{~B} N \mathrm{NMR}$ (96 $\mathrm{MHz}$, Chloroform- $d, \delta \mathrm{ppm}$ ) 31.2. The spectroscopic data match with those reported in the literature. ${ }^{38}$

\section{2-(5-Methoxy-4-methyl-2-(prop-1-en-2-yl)phenyl)-4,4,5,5- tetramethyl-1,3,2-dioxaborolane (4d)}

White solid, $184 \mathrm{mg}, 64 \%$; mp 56-57 ${ }^{\circ} \mathrm{C}$; ${ }^{1} \mathrm{H}$ NMR (300 MHz, Chloroform-d, $\delta \mathrm{ppm}) 7.06(\mathrm{~s}, 1 \mathrm{H}), 7.02(\mathrm{~d}, J=0.9 \mathrm{~Hz}, 1 \mathrm{H})$, 5.02-4.99 (m, 1H), 4.84-4.80 (m, 1H), $3.86(\mathrm{~s}, 3 \mathrm{H}), 2.21(\mathrm{~s}, 3 \mathrm{H})$, $2.11(\mathrm{~s}, 1 \mathrm{H}), 1.32$ (s, 12H). ${ }^{13} \mathrm{C}$ NMR (75 MHz, Chloroform- $d, \delta$ ppm) 156.1, 147.3, 142.3, 129.9, 128.5, 115.2, 113.5, 83.5, $55.4,24.75,24.7,16.3$ (the carbon $\alpha$ to boron was not found). ${ }^{11} \mathrm{~B}$ NMR (128 MHz, Chloroform- $d, \delta \mathrm{ppm}$ ) 31.5. HRMS (ESI+) calcd. for $[\mathrm{M}+\mathrm{Na}]^{+}\left(\mathrm{C}_{17} \mathrm{H}_{25} \mathrm{O}_{3}{ }^{11} \mathrm{BNa}\right): 311.1788$, found : 311.1789 .

\section{4,4,5,5-Tetramethyl-2-(4-nitro-2-(prop-1-en-2-yl)phenyl)- 1,3,2-dioxaborolane (4e)}

White solid, $118 \mathrm{mg}, 41 \%$; mp 78-79 ${ }^{\circ} \mathrm{C}$; ${ }^{1} \mathrm{H}$ NMR $(400 \mathrm{MHz}$, Chloroform- $d, \delta$ ppm) 8.07-8.03 (m, $2 \mathrm{H}), 7.75(\mathrm{~d}, J=8.9 \mathrm{~Hz}$, $1 \mathrm{H}$ ), 5.17 (quint, $J=1.5 \mathrm{~Hz}, 1 \mathrm{H}$ ), 4.96-4.94 (m, 1H), 2.16 (dd, $J=$ 1.5, $0.9 \mathrm{~Hz}, 3 \mathrm{H}), 1.34$ (s, 12H). ${ }^{13} \mathrm{C}$ NMR $(101 \mathrm{MHz}$, Chloroformd, $\delta$ ppm) 150.9, 148.9, 145.5, 135.1, 121.5, 120.6, 116.2, 84.4, 24.7, 24.1 (the carbon $\alpha$ to boron was not found). ${ }^{11} \mathrm{~B}$ NMR (128 MHz, Chloroform-d, $\delta \mathrm{ppm}$ ) 30.4. HRMS (ESI+) calcd. for $[\mathrm{M}+\mathrm{Na}]^{+}\left(\mathrm{C}_{15} \mathrm{H}_{20} \mathrm{NO}_{4}{ }^{11} \mathrm{BNa}\right): 312.1377$, found 312.1375 .

\section{4,4,5,5-Tetramethyl-2-(4-chloro-2-(prop-1-en-2-yl)phenyl)- 1,3,2-dioxaborolane (4f)}

Colorless oil, $173 \mathrm{mg}, 62 \% ;{ }^{1} \mathrm{H}$ NMR (400 MHz, Chloroform- $d, \delta$ ppm) 7.62-7.58 (m, $1 \mathrm{H}), 7.26-7.22(\mathrm{~m}, 2 \mathrm{H}), 5.11$ (quint, $J=1.6$ $\mathrm{Hz}, 1 \mathrm{H}), 4.92-4.90(\mathrm{~m}, 1 \mathrm{H}), 2.14(\mathrm{t}, J=1.2 \mathrm{~Hz}, 3 \mathrm{H}), 1.34(\mathrm{~s}, 12 \mathrm{H})$. ${ }^{13} \mathrm{C}$ NMR (101 MHz, Chloroform- $d, \delta$ ppm) 151.5, 146.4, 136.0, $135.9,127.3,126.2,115.0,83.8,24.7,24.4$ (The carbon $\alpha$ to boron was not found). ${ }^{11} \mathrm{~B}$ NMR (128 MHz, Chloroform- $d, \delta$ ppm) 31.4. HRMS $\left(\mathrm{ESI}^{+}\right)$calcd. for $[\mathrm{M}+\mathrm{Na}]^{+}\left(\mathrm{C}_{15} \mathrm{H}_{20}{ }^{35} \mathrm{ClO}_{2}{ }^{11} \mathrm{BNa}\right)$ : 301.1137 , found 301.1142 .

\section{General procedure for the synthesis of B-MIDA derivatives (6)}

A flask equipped with a stirring bar was charged with boronic acid (3 mmol), $N$-methyliminodiacetic acid (441 $\mathrm{mg}, 3 \mathrm{mmol}$ ) and toluene/DMSO (15 mL/3 mL). The flask was then equipped with a Dean-Stark trap and a reflux condenser, and heated at reflux for 20 hours. The reaction mixture was allowed to cool to $\mathrm{rt}$ and the solvents were removed by distillation. Dichloromethane was added to the resulting crude that led to the precipitation of the desired product. The solid was collected by filtration and rinsed with cold dichloromethane.

\section{2-(2-Bromophenyl)-6-methyl-1,3,6,2-dioxazaborocane-4,8- dione (6a)}

White solid, $197 \mathrm{mg}, 88 \%$; mp 206-8 ${ }^{\circ} \mathrm{C}$; ${ }^{1} \mathrm{H}$ NMR $(300 \mathrm{MHz}$, Acetonitrile- $\left.d_{3}, \delta \mathrm{ppm}\right) 7.69$ (dd, $\left.J=7.5,2.0 \mathrm{~Hz}, 1 \mathrm{H}\right), 7.63(\mathrm{dd}, J$ $=7.9,1.2 \mathrm{~Hz}, 1 \mathrm{H}$ ), 7.41 (ddd, $J=7.5,7.3,1.2 \mathrm{~Hz}, 1 \mathrm{H}$ ), 7.32 (ddd, $J=7.9,7.3,2.0 \mathrm{~Hz}, 1 \mathrm{H}), 4.18(\mathrm{~d}, J=17.3 \mathrm{~Hz}, 2 \mathrm{H}), 4.06(\mathrm{~d}, J=$ $17.3 \mathrm{~Hz}, 2 \mathrm{H}), 2.74(\mathrm{~s}, 3 \mathrm{H}) .{ }^{13} \mathrm{C}$ NMR $\left(101 \mathrm{MHz}\right.$, Acetonitrile- $d_{3}, \delta$ ppm) $169.5,137.3,134.5,132.3,128.6,127.9,65.2,49.5$ (the carbon $\alpha$ to boron was not found). ${ }^{11} \mathrm{~B} N M R$ (96 $\mathrm{MHz}$, Acetonitrile- $\left.d_{3}, \delta \mathrm{ppm}\right)$ 11.5. The spectroscopic data match with those reported in the literature. ${ }^{40}$

\section{2-(2-Bromo-6-fluorophenyl)-6-methyl-1,3,6,2 dioxazaborocane-4,8-dione $(6 \mathrm{~g})$}

White solid, $851 \mathrm{mg}, 86 \%$; $\mathrm{mp} 214-216{ }^{\circ} \mathrm{C}$; ${ }^{1} \mathrm{H} \mathrm{NMR}(300 \mathrm{MHz}$, Acetonitrile- $d_{3}, \delta$ ppm) 7.49 (ddd, $J=8.0,1.0,0.8 \mathrm{~Hz}, 1 \mathrm{H}$ ), 7.31 (ddd, $J=8.2,8.1,6.2 \mathrm{~Hz}, 1 \mathrm{H}$ ), 7.10 (ddd, $J=11.0,8.2,1.1 \mathrm{~Hz}$, 
$1 \mathrm{H}), 4.13(\mathrm{~d}, J=17.1,2 \mathrm{H}), 3.97(\mathrm{~d}, J=17.1,2 \mathrm{H}), 2.78(\mathrm{~s}, 3 \mathrm{H}) .{ }^{13} \mathrm{C}$ NMR $\left(75 \mathrm{MHz}\right.$, Acetonitrile- $\left.d_{3}, \delta \mathrm{ppm}\right) 169.3,168.1(\mathrm{~d}, J=$ $247.1 \mathrm{~Hz}$ ), 133.5 (d, J=11.0 Hz), 131.7 (d, J=3.1 Hz), 129.1 (d, $J=9.4 \mathrm{~Hz}$ ), 116.1 (d, $J=27.7 \mathrm{~Hz}$ ), 64.3, 64.25, 49.0 (The carbon $\alpha$ to boron was not found). ${ }^{19} \mathrm{~F}$ NMR (282 MHz, Acetonitrile- $d_{3}$, $\delta \mathrm{ppm})$-99.3. ${ }^{11} \mathrm{~B} \mathrm{NMR}\left(128 \mathrm{MHz}\right.$, Acetonitrile- $\left.d_{3}, \delta \mathrm{ppm}\right) 11.1$. The spectroscopic data match with those reported in the literature. ${ }^{40}$

\section{2-(2-Bromo-4-methylphenyl)-6-methyl-1,3,6,2- dioxazaborocane-4,8-dione (6h)}

White solid, $851 \mathrm{mg}, 87 \%$; mp 230-232 ${ }^{\circ} \mathrm{C}$; ${ }^{1} \mathrm{H}$ NMR $(300 \mathrm{MHz}$, Acetonitrile- $\left.d_{3}, \delta \mathrm{ppm}\right) 7.54(\mathrm{~d}, J=7.7 \mathrm{~Hz}, 1 \mathrm{H}), 7.47(\mathrm{~s}, 1 \mathrm{H})$, $7.20(\mathrm{~d}, J=8.0 \mathrm{~Hz}, 1 \mathrm{H}), 4.12(\mathrm{~d}, J=17.4 \mathrm{~Hz}, 2 \mathrm{H}), 4.01(\mathrm{~d}, J=$ $17.3 \mathrm{~Hz}, 2 \mathrm{H}), 2.69(\mathrm{~s}, 3 \mathrm{H}), 2.32(\mathrm{~s}, 3 \mathrm{H}) .{ }^{13} \mathrm{C} \mathrm{NMR}(75 \mathrm{MHz}$, Acetonitrile- $\left.d_{3}, \delta \mathrm{ppm}\right) 169.5,142.9,137.2,135.0,128.8$, $128.5,65.2,49.4,20.7$ (the carbon $\alpha$ to boron was not found). ${ }^{11} \mathrm{~B}$ NMR (96 MHz, Acetonitrile- $d_{3}, \delta \mathrm{ppm}$ ) 11.6. HRMS (ESI ${ }^{+}$) calcd. for $[\mathrm{M}+\mathrm{Na}]^{+}\left(\mathrm{C}_{12} \mathrm{H}_{13} \mathrm{NO}_{4}{ }^{79} \mathrm{Br}^{11} \mathrm{BNa}\right)$ : 348.0013, found: 348.0013 .

\section{$N$-(3-bromo-4-(6-methyl-4,8-dioxo-1,3,6,2-dioxazaborocan-2-} yl)phenyl)acetamide (6i)

White solid, mp 194-198 ${ }^{\circ} \mathrm{C}, 686 \mathrm{mg}, 62 \%$. RMN ${ }^{1} \mathrm{H}(400 \mathrm{MHz}$, Acetonitrile- $\left.d_{3}, \delta \mathrm{ppm}\right)$ 8.57-8.41 (m, $\left.1 \mathrm{H}\right), 7.77(\mathrm{dd}, J=8.6,2.8$ $\mathrm{Hz}, 1 \mathrm{H}$ ), 7.67 (d, $J=2.8 \mathrm{~Hz}, 1 \mathrm{H}), 7.51(\mathrm{~d}, J=8.6 \mathrm{~Hz}, 1 \mathrm{H}), 4.14$ (d, $J=17.4 \mathrm{~Hz}, 2 \mathrm{H}), 4.03(\mathrm{~d}, J=17.4 \mathrm{~Hz}, 2 \mathrm{H}), 2.76(\mathrm{~s}, 3 \mathrm{H}), 2.06(\mathrm{~s}$, $3 \mathrm{H}) . \mathrm{RMN}^{13} \mathrm{C}\left(101 \mathrm{MHz}\right.$, Acetonitrile- $\left.d_{3}, \delta \mathrm{ppm}\right) 169.7,169.5$, 139.2, 134.9, 127.7, 123.0, 121.7, 65.1, 49.5, 24.3. RMN111 B (96 $\mathrm{MHz}$, Acetonitrile-d3, $\delta \mathrm{ppm})$ 11.4. The spectroscopic data match with those reported in the literature. ${ }^{25}$

\section{General procedure for the synthesis of boronates $4 a, g-i$ from B-MIDA derivatives 6}

A oven-dried Schlenk tube equipped with a stirring bar was charged under argon with 2-bromoarylboronic acid MIDA ester 6 (2 mmol), 4,4,5,5-tetramethyl-2-(prop-1-en)-2-yl)-1,3,2dioxaborolane (500 mg, $3 \mathrm{mmol}, 1.5$ equiv.), $\mathrm{PdCl}_{2}$ (dppf) (58 $\mathrm{mg}, 0.08 \mathrm{mmol}, 4 \mathrm{~mol} \%)$ and $\mathrm{K}_{3} \mathrm{PO}_{4}(1.24 \mathrm{~g}, 6 \mathrm{mmol})$, anhydrous THF $(8 \mathrm{~mL})$ and water $(180 \mu \mathrm{L}, 10 \mathrm{mmol})$. The reaction mixture was then heated in a oil bath for $20 \mathrm{~h}$ at $90^{\circ} \mathrm{C}$ for $\mathbf{4 a}, \mathbf{g}-\mathbf{h}$ and at $40^{\circ} \mathrm{C}$ for $\mathbf{4 i}$. The mixture was allowed to cool to room temperature, diluted with $\mathrm{H}_{2} \mathrm{O}(4 \mathrm{~mL})$ and extracted with EtOAc $(3 \times 10 \mathrm{~mL})$. The combined organic layers dried over $\mathrm{MgSO}_{4}$, filtered and concentrated in vacuo. The crude product was purified with column chromatography on silica gel (cyclohexane/AcOEt: 10/4) or Kugelrohr distillation.

\section{2-(2-Fluoro-6-(prop-1-en-2-yl)phenyl)-4,4,5,5-tetramethyl- 1,3,2-dioxaborolane (4g)}

Colorless oil, $183 \mathrm{mg}, 35 \%$ yield; bp 75-80/0.01 mm Hg. ${ }^{1} \mathrm{H}$ NMR (300 MHz Chloroform- $d, \delta$ ppm) 7.27 (td, $J=7.8,6.2 \mathrm{~Hz}$, $1 \mathrm{H}), 7.02(\mathrm{~d}, J=7.6 \mathrm{~Hz}, 1 \mathrm{H}), 6.90(\mathrm{t}, J=8.4 \mathrm{~Hz}, 1 \mathrm{H}), 5.08(\mathrm{q}, J=$ $1.6 \mathrm{~Hz}, 1 \mathrm{H}), 5.04-4.98(\mathrm{~m}, 1 \mathrm{H}), 2.12(\mathrm{t}, J=0.8 \mathrm{~Hz}, 3 \mathrm{H}), 1.35(\mathrm{~s}$, 12H). ${ }^{13} \mathrm{C}$ NMR (101 MHz, Chloroform- $\left.d, \delta \mathrm{ppm}\right) 165.6$ (d, $J=$ $242.2 \mathrm{~Hz}$ ), 149.9 (d, $J=8.5 \mathrm{~Hz}), 146.2$ (d, $J=1.9 \mathrm{~Hz}), 130.7$ (d, $J$ $=9.0 \mathrm{~Hz}), 122.3(\mathrm{~d}, J=2.8 \mathrm{~Hz}), 115.3,113.2(\mathrm{~d}, J=23.9 \mathrm{~Hz})$,
84.3, 24.8, 24.0 (the carbon $\alpha$ to boron was not found). ${ }^{11} \mathrm{~B}$ NMR (96 MHz, Chloroform-d, $\delta$ ppm) 31.1. ${ }^{19} \mathrm{~F}$ NMR (282 MHz, Chloroform- $d, \delta \mathrm{ppm})$-105.65. HRMS $\left(\mathrm{ESI}^{+}\right)$calcd. for $[\mathrm{M}+\mathrm{Na}]^{+}$ $\left(\mathrm{C}_{15} \mathrm{H}_{20} \mathrm{O}_{2} \mathrm{~F}^{11} \mathrm{BNa}\right)$ : 285.1432, found: 285.1429.

\section{4,4,5,5-Tetramethyl-2-(4-methyl-2-(prop-1-en-2-yl)phenyl)- 1,3,2-dioxaborolane (4h)}

Colorless oil, $258 \mathrm{mg}, 50 \%$; ${ }^{1} \mathrm{H}$ NMR (300 MHz, Chloroform- $d, \delta$ ppm) $7.57(\mathrm{~d}, J=7.4 \mathrm{~Hz}, 1 \mathrm{H}), 7.12-7.03(\mathrm{~m}, 2 \mathrm{H}), 5.07$ (dq, $J=$ 2.9, $1.5 \mathrm{~Hz}, 1 \mathrm{H}), 4.88(\mathrm{dq}, J=2.9,0.9 \mathrm{~Hz}, 1 \mathrm{H}), 2.36(\mathrm{~s}, 3 \mathrm{H}), 2.15$ $(\mathrm{dd}, J=1.5,0.9 \mathrm{~Hz}, 3 \mathrm{H}), 1.34(\mathrm{~s}, 12 \mathrm{H}) .{ }^{13} \mathrm{C} \mathrm{NMR}(75 \mathrm{MHz}$, Chloroform- $d, \delta$ ppm) 150.2, 147.9, 140.0, 134.9, 128.1, 127.1, $113.9,83.5,24.9,24.8,21.6$ (the carbon $\alpha$ to boron was not found). ${ }^{11} \mathrm{~B}$ NMR (128 MHz, Chloroform- $d, \delta \mathrm{ppm}$ ) 31.0. HRMS $\left(E S I^{+}\right)$calc. for $[\mathrm{M}+\mathrm{Na}]^{+}\left(\mathrm{C}_{16} \mathrm{H}_{23} \mathrm{O}_{2}{ }^{11} \mathrm{BNa}\right): 281.1683$, found: 281.1688 .

\section{N-(3-(prop-1-en-2-yl)-4-(4,4,5,5-tetramethyl-1,3,2- dioxaborolan-2-yl)phenyl)acetamide (4i)}

Colorless oil, $325 \mathrm{mg}, 54 \%$ yield. ${ }^{1} \mathrm{H}$ NMR $(300 \mathrm{MHz}$, Acetonitrile- $\left.d_{3}, \delta \mathrm{ppm}\right) 8.28(\mathrm{br} \mathrm{s}, 1 \mathrm{H}), 7.68(\mathrm{~d}, J=2.4 \mathrm{~Hz}, 1 \mathrm{H})$, 7.61 (dd, $J=8.4,2.4 \mathrm{~Hz}, 1 \mathrm{H}), 7.22(\mathrm{~d}, J=8.3 \mathrm{~Hz}, 1 \mathrm{H}), 5.09-5.01$ $(\mathrm{m}, 1 \mathrm{H}), 4.84(\mathrm{dq}, J=1.8,0.9 \mathrm{~Hz}, 1 \mathrm{H}), 2.17(\mathrm{~s}, 3 \mathrm{H}), 2.10(\mathrm{dd}, J=$ 1.5, $0.9 \mathrm{~Hz}, 3 \mathrm{H}) .{ }^{13} \mathrm{C} \mathrm{NMR}\left(101 \mathrm{MHz}, \mathrm{CD}_{3} \mathrm{CN}, \delta \mathrm{ppm}\right) 168.5$, $147.0,144.5,137.4,127.6,124.9,120.9,113.7,83.7,24.0$, 23.6. ${ }^{11} \mathrm{~B}$ NMR (128 MHz, Chloroform-d, $\left.\delta \mathrm{ppm}\right)$ 31.2. HRMS $\left(\mathrm{ESI}^{+}\right)$calc. for $[\mathrm{M}+\mathrm{Na}]^{+}\left(\mathrm{C}_{17} \mathrm{H}_{24} \mathrm{NO}_{3}{ }^{11} \mathrm{BNa}\right)$ : 324.1741, found: 324.1740 .

\section{General procedure for the ene reactions}

To pre-dried $\left(18 \mathrm{~h}, 100^{\circ} \mathrm{C}\right.$ under vacuum) scandium triflate (49 $\mathrm{mg}, 0.1 \mathrm{mmol}, 10 \mathrm{~mol} \%)$ in anhydrous DCM $(10 \mathrm{~mL})$, was added $\alpha$-methylstyrenes $4(1 \mathrm{mmol})$. The reaction mixture was cooled at $0-5^{\circ} \mathrm{C}$. Ethyl glyoxylate $(288 \mu \mathrm{L}, 1.5 \mathrm{mmol}, 50 \%$ in toluene, 1.5 equiv.) was added dropwise. ${ }^{41}$ The mixture was stirred overnight at room temperature. The resulting solution is washed twice with brine. The organic layer was dried over anhydrous sodium sulfate, filtered, and concentrated under reduced pressure. The crude is directly engaged in the following oxidation step. A small amount was purified for spectral characterization using preparative thin-layer chromatography on silica gel (ethyl acetate/cyclohexane: 3/1).

Ethyl 2-hydroxy-4-(2-(4,4,5,5-tetramethyl-1,3,2-dioxaborolan2-yl)phenyl)pent-4-enoate (7a)

Colorless oil, 57\% from 4a: $\mathrm{RMN}^{1} \mathrm{H}(300 \mathrm{MHz}$, Chloroform- $d, \delta$ ppm) 7.74-7.69 (m, 1H), 7.46-7.38 (m, $1 \mathrm{H})$, 7.34-7.27 (m, $2 \mathrm{H})$, 5.33-5.29 (m, $1 \mathrm{H}), 5.01(\mathrm{~d}, J=1.8 \mathrm{~Hz}, 1 \mathrm{H}), 4.64(\mathrm{~d}, J=5.4 \mathrm{~Hz}$, $1 \mathrm{H}$ ), 4.19 (ddd, $J=8.8,5.4,3.3 \mathrm{~Hz}, 1 \mathrm{H}$ ), 4.08-3.97 (m, $2 \mathrm{H}$ ), 3.10 (ddd, $J=13.6,3.3,1.3 \mathrm{~Hz}, 1 \mathrm{H}), 2.87$ (dd, $J=13.6,8.8 \mathrm{~Hz}, 1 \mathrm{H}$ ), $1.360(\mathrm{~s}, 6 \mathrm{H}), 1.355(\mathrm{~s}, 6 \mathrm{H}), 1.21(\mathrm{t}, J=7.1 \mathrm{~Hz}, 3 \mathrm{H}) . \mathrm{RMN}^{13} \mathrm{C}(75$ $\mathrm{MHz}$, Chloroform- $d, \delta \mathrm{ppm})$ 173.0, 146.9, 145.3, 135.1, 130.2, $127.0,126.8,120.4,84.4,68.8,60.9,43.6,24.6,24.3,14.1$ (the carbon $\alpha$ to boron was not found). ${ }^{11} \mathrm{~B}$ NMR $(128 \mathrm{MHz}$, Chloroform- $d, \delta \mathrm{ppm})$ 31.1. HRMS $\left(\mathrm{ESI}^{+}\right)$calc. for $[\mathrm{M}+\mathrm{Na}]^{+}$ $\left(\mathrm{C}_{19} \mathrm{H}_{27} \mathrm{O}_{5}{ }^{11} \mathrm{BNa}\right): 269.0956$, found: 269.0958 . 
Ethyl 1-hydroxy-5-methylene-1,3,4,5-tetrahydrobenzo[c][1,2] oxaborepine-3-carboxylate (8a)

Colorless oil, 75\% from 7a: ${ }^{1} \mathrm{H}$ NMR $(300 \mathrm{MHz}$, Chloroform- $d, \delta$ ppm) $8.02(\mathrm{~d}, J=7.4 \mathrm{~Hz}, 1 \mathrm{H}), 7.52-7.30(\mathrm{~m}, 3 \mathrm{H}), 5.34(\mathrm{~d}, J=1.0$ $\mathrm{Hz}, 1 \mathrm{H}), 5.16(\mathrm{q}, J=1.0 \mathrm{~Hz}, 1 \mathrm{H}), 4.82(\mathrm{dd}, J=6.7,4.5 \mathrm{~Hz}, 1 \mathrm{H})$, $4.54(\mathrm{br} \mathrm{s}, 1 \mathrm{H}), 4.22(\mathrm{q}, J=7.1 \mathrm{~Hz}, 2 \mathrm{H}), 3.11-3.06(\mathrm{~m}, 2 \mathrm{H}), 1.28$ $(\mathrm{t}, J=7.1 \mathrm{~Hz}, 3 \mathrm{H}) .{ }^{13} \mathrm{C}$ NMR (101 MHz, Chloroform- $\left.d, \delta \mathrm{ppm}\right)$ $171.3,146.5,145.8,136.0,131.3,127.2,126.9,117.4,74.2$, $61.4,41.2,14.2$ (the carbon $\alpha$ to boron was not found). ${ }^{11} \mathrm{~B}$ NMR (128 MHz, Chloroform-d, $\delta$ ppm) 28.8. HRMS (ESI+) calc. for $[\mathrm{M}+\mathrm{Na}]^{+}\left(\mathrm{C}_{13} \mathrm{H}_{15} \mathrm{O}_{4}{ }^{11} \mathrm{BNa}\right)$ : 281.1683, found: 281.1688 .

\section{General procedure for the oxidation of the ene products 7}

To a solution of the crude boronate 7 obtained from 4 (1 $\mathrm{mmol})$ in $\mathrm{THF} / \mathrm{H}_{2} \mathrm{O}(10 / 5 \mathrm{~mL})$ was added sodium perborate tetrahydrate ( $411 \mathrm{mg}, 3 \mathrm{mmol}$ ). The mixture was stirred at room temperature overnight. THF was removed under reduced pressure and the residue was stirred with sat. $\mathrm{NH}_{4} \mathrm{Cl}$ $(5 \mathrm{~mL})$ and methylene chloride $(30 \mathrm{~mL})$. The organics layers were separated, dried over $\mathrm{MgSO}_{4}$ and concentrated. The residue was purified by column chromatography on silica gel (cyclohexane/ethyl acetate: 6/4).

\section{Ethyl 2-hydroxy-4-(2-hydroxyphenyl)pent-4-enoate (9a)}

Colorless oil, $137 \mathrm{mg}, 58 \%$; ${ }^{1} \mathrm{H}$ NMR (400 MHz, Chloroform- $d, \delta$ ppm) 7.23-7.13 (m, 1H), 7.08 (dd, $J=7.6,1.7 \mathrm{~Hz}, 1 \mathrm{H}), 6.94$ (dd, $J=8.1,1.2 \mathrm{~Hz}, 1 \mathrm{H}), 6.91(\mathrm{dd}, J=7.4,1.2 \mathrm{~Hz}, 1 \mathrm{H}), 6.43(\mathrm{~s}, 1 \mathrm{H})$, $5.44(\mathrm{dt}, J=1.6,0.8 \mathrm{~Hz}, 1 \mathrm{H}), 5.27(\mathrm{dt}, J=1.6,0.8 \mathrm{~Hz}, 1 \mathrm{H}), 4.24-$ $4.07(\mathrm{~m}, 3 \mathrm{H}), 3.12(\mathrm{~d}, J=4.9 \mathrm{~Hz}, 1 \mathrm{H}), 2.97(\mathrm{dd}, J=14.1,4.9$ $\mathrm{Hz} 1 \mathrm{H}), 2.74$ (ddd, $J=14.1,8.6,0.9 \mathrm{~Hz}, 1 \mathrm{H}), 1.25(\mathrm{t}, J=7.1 \mathrm{~Hz}$, $3 \mathrm{H}) .{ }^{13} \mathrm{C}$ NMR (101 MHz, Chloroform-d, $\left.\delta \mathrm{ppm}\right) 174.1,153.1$, 140.9, 129.1, 128.7, 127.7, 120.0, 116.2, 68.8, 62.0, 42.1, 14.1. HRMS $\left(\mathrm{ESI}^{+}\right)$calculated for $[\mathrm{M}+\mathrm{Na}]^{+}\left(\mathrm{C}_{13} \mathrm{H}_{16} \mathrm{O}_{4} \mathrm{Na}\right)$ : 259.0940, found: 259.0943.

Ethyl 4-(4-fluoro-2-hydroxyphenyl)-2-hydroxypent-4-enoate (9b)

Colorless oil, $86 \mathrm{mg}, 34 \%$; ${ }^{1} \mathrm{H}$ NMR (400 MHz, Chloroform- $d, \delta$ ppm) 7.02 (dd, $J=8.5,6.6 \mathrm{~Hz}, 1 \mathrm{H}), 6.73(\mathrm{br} \mathrm{s}, 1 \mathrm{H}), 6.64(\mathrm{dd}, J=$ $10.3,2.6 \mathrm{~Hz}, 1 \mathrm{H}$ ), 6.59 (ddd, $J=8.5,8.4,2.6 \mathrm{~Hz}, 1 \mathrm{H}$ ), 5.45-5.43 $(\mathrm{m}, 1 \mathrm{H}), 5.26-5.23(\mathrm{~m}, 1 \mathrm{H}), 4.30-4.11(\mathrm{~m}, 3 \mathrm{H}), 3.13(\mathrm{~d}, J=4.6$ $\mathrm{Hz}, 1 \mathrm{H}$ ), 2.91 (dd, $J=14.1,4.5 \mathrm{~Hz}, 1 \mathrm{H}), 2.70$ (ddd, $J=14.1,8.8$, $0.9 \mathrm{~Hz}, 1 \mathrm{H}), 1.27(\mathrm{t}, J=7.1 \mathrm{~Hz}, 3 \mathrm{H}) .{ }^{13} \mathrm{C} \mathrm{NMR}(101 \mathrm{MHz}$, Chloroform-d, $\delta$ ppm) 174.1, $163.2(\mathrm{~d}, J=245.2 \mathrm{~Hz}), 154.7$ (d, J $=12.3 \mathrm{~Hz}), 140.3,129.6(\mathrm{~d}, J=10.0 \mathrm{~Hz}), 123.9(\mathrm{~d}, J=3.3 \mathrm{~Hz})$, 120.6, 107.1 (d, $J=21.6 \mathrm{~Hz}), 103.8(\mathrm{~d}, J=24.6 \mathrm{~Hz}), 69.0,62.3$, 42.1, 14.2. ${ }^{19} \mathrm{~F}$ NMR (376 MHz, Chloroform-d, $\delta \mathrm{ppm}$ ) -112.8. HRMS $\left(\mathrm{ESI}^{+}\right)$calculated for $[\mathrm{M}+\mathrm{Na}]^{+}\left(\mathrm{C}_{13} \mathrm{H}_{15} \mathrm{FO}_{4} \mathrm{Na}\right): 277.0847$, found: 277.0847.

\section{Ethyl 2-hydroxy-4-(2-hydroxy-4-methoxyphenyl)pent-4- enoate (9c)}

Colorless oil, $138 \mathrm{mg}, 52 \% .{ }^{1} \mathrm{H}$ NMR (300 MHz, Chloroform- $d, \delta$ ppm) $6.98(\mathrm{~d}, J=8.3 \mathrm{~Hz}, 1 \mathrm{H}), 6.53(\mathrm{br} \mathrm{s}, 1 \mathrm{H}), 6.49(\mathrm{~d}, J=2.4 \mathrm{~Hz}$, $1 \mathrm{H}), 6.45(\mathrm{dd}, J=8.3,2.5 \mathrm{~Hz}, 1 \mathrm{H}), 5.43-5.40(\mathrm{~m}, 1 \mathrm{H}), 5.25-5.22$ $(\mathrm{m}, 1 \mathrm{H}), 4.24-4.06(\mathrm{~m}, 3 \mathrm{H}), 3.77(\mathrm{~s}, 3 \mathrm{H}), 3.15(\mathrm{~d}, J=5.0 \mathrm{~Hz}, 1 \mathrm{H})$, 2.91 (dddd, $J=14.1,4.6,1.5,0.7 \mathrm{~Hz}, 1 \mathrm{H}$ ), 2.71 (ddd, $J=14.1$, 8.5, $0.9 \mathrm{~Hz}, 1 \mathrm{H}), 1.26(\mathrm{t}, J=7.1 \mathrm{~Hz}, 3 \mathrm{H}) .{ }^{13} \mathrm{C} \mathrm{NMR}(75 \mathrm{MHz}$, Chloroform- $d, \delta$ ppm) 174.3, 160.5, 154.4, 140.9, 129.4, 120.3, 119.7, 106.5, 101.6, 69.1, 62.2, 55.4, 42.3, 14.2. HRMS (ESI+) calculated for $[\mathrm{M}+\mathrm{Na}]^{+}\left(\mathrm{C}_{14} \mathrm{H}_{18} \mathrm{O}_{5} \mathrm{Na}\right): 289.1046$, found: 289.1049 .

\section{Ethyl 2-hydroxy-4-(2-hydroxy-4-methoxy-5-methylphenyl) pent-4-enoate (9d)}

Colorless oil, $151 \mathrm{mg}, 54 \%$; ${ }^{1} \mathrm{H}$ NMR (400 MHz, Chloroform- $d$, $\delta$ ppm) $6.84(\mathrm{~d}, J=1.0 \mathrm{~Hz}, 1 \mathrm{H}), 6.47(\mathrm{~s}, 1 \mathrm{H}), 6.36(\mathrm{br} \mathrm{s}, 1 \mathrm{H}), 5.41-$ $5.39(\mathrm{~m}, 1 \mathrm{H}), 5.23-5.21(\mathrm{~m}, 1 \mathrm{H}), 4.25-4.10(\mathrm{~m}, 3 \mathrm{H}), 3.81(\mathrm{~s}, 3 \mathrm{H})$, 3.11-3.05 (m, 1H), 2.93 (dd, $J=13.8,4.7 \mathrm{~Hz}, 1 \mathrm{H}), 2.71$ (ddd, $J=$ 14.0, 8.4, 0.9 Hz, 1H), 2.12 (s, 3H), 1.26 (t, J = 7.2 Hz, 3H). ${ }^{13} \mathrm{C}$ NMR (101 MHz, Chloroform-d, $\delta$ ppm) 174.2, 158.1, 152.2, 140.9, 129.8, 119.3, 118.7, 117.9, 98.9, 68.9, 62.0, 55.4, 42.3, 15.3, 14.1. HRMS $\left(\mathrm{ESI}^{+}\right)$calculated for $[\mathrm{M}+\mathrm{Na}]^{+}\left(\mathrm{C}_{15} \mathrm{H}_{20} \mathrm{O} \mathrm{O}_{5} \mathrm{Na}\right)$ : 303.1203, found: 303.1204 .

Ethyl 4-(5-chloro-2-hydroxyphenyl)-2-hydroxypent-4-enoate (9e)

Colorless oil, $133 \mathrm{mg}$, $49 \%$; ${ }^{1} \mathrm{H}$ NMR (300 MHz, Chloroform- $d, \delta$ ppm) 7.15 (dd, $J=8.7,2.6 \mathrm{~Hz}, 1 \mathrm{H}$ ), $7.07(\mathrm{~d}, J=2.6 \mathrm{~Hz}, 1 \mathrm{H}), 6.87$ (d, $J=8.7 \mathrm{~Hz}, 2 \mathrm{H}), 6.58(\mathrm{br} \mathrm{s}, 1 \mathrm{H}), 5.47-5.49(\mathrm{~m}, 1 \mathrm{H}), 5.30-5.28$ $(\mathrm{m}, 1 \mathrm{H}), 4.26-4.13(\mathrm{~m}, 3 \mathrm{H}), 3.19(\mathrm{br} \mathrm{s}, 1 \mathrm{H}), 2.94$ (ddt, $J=14.2$, 4.7, $1.1 \mathrm{~Hz}, 1 \mathrm{H}$ ), 2.73 (ddd, $J=14.2,8.7,1.0 \mathrm{~Hz}, 1 \mathrm{H}$ ), 1.30 (t, $J=$ $7.2 \mathrm{~Hz}, 3 \mathrm{H}) .{ }^{13} \mathrm{C}$ NMR (101 MHz, Chloroform-d, $\left.\delta \mathrm{ppm}\right) \delta 173.9$, $151.9,139.9,129.2,128.9,128.3,124.7,120.9,117.7,68.8$, $62.3,41.6,14.1$. HRMS $\left(E S I^{+}\right)$calculated for $[\mathrm{M}+\mathrm{H}]^{+}\left(\mathrm{C}_{13} \mathrm{H}_{16} \mathrm{O}_{4}\right)$ : 271.0732, found: 271.0731 .

\section{Ethyl 4-(3-fluororo-2-hydroxyphenyl)-2-hydroxypent-4- enoate (9g)}

Colorless oil, 104 mg, $41 \%$; ${ }^{1} \mathrm{H}$ NMR (400 MHz, Chloroform- $d$, $\delta$ ppm) 7.00 (ddd, $J=10.5,8.1,1.6 \mathrm{~Hz}, 1 \mathrm{H}$ ), 6.89 (dd, $J=7.8,1.6$ $\mathrm{Hz}, 1 \mathrm{H}), 6.80$ (ddd, $J=8.1,7.9,5.0 \mathrm{~Hz}, 1 \mathrm{H}), 5.40-5.42(\mathrm{~m}, 1 \mathrm{H})$, 5.29-5.27 (m, 1H), 4.23-4.06 (m, 3H), 3.00 (dddd, $J=14.2,4.5$, $1.4,0.8 \mathrm{~Hz}, 1 \mathrm{H}$ ), 2.78 (ddd, $J=14.2,8.4,0.9 \mathrm{~Hz}, 1 \mathrm{H}), 1.25(\mathrm{t}, J=$ $7.2 \mathrm{~Hz}, 3 \mathrm{H}) .{ }^{13} \mathrm{C}$ NMR (101 MHz, Chloroform-d, $\left.\delta \mathrm{ppm}\right)$ 174.1, $151.5(\mathrm{~d}, J=241.3 \mathrm{~Hz}), 141.5(\mathrm{~d}, J=12.7 \mathrm{~Hz}), 140.6(\mathrm{~d}, J=2.8$ $\mathrm{Hz}), 130.5(\mathrm{~d}, J=1.8 \mathrm{~Hz}), 124.5(\mathrm{~d}, J=3.4 \mathrm{~Hz}), 120.4,119.9$ (d, $J$ $=7.4 \mathrm{~Hz}), 115.2(\mathrm{~d}, J=18.4 \mathrm{~Hz}), 69.2,62.1,41.6,14.2 .{ }^{19} \mathrm{~F} \mathrm{NMR}$ (376 MHz, Chloroform-d, $\delta \mathrm{ppm}$ ) -137.9. HRMS (ESI $\left.{ }^{+}\right)$ calculated for $[\mathrm{M}+\mathrm{Na}]^{+}\left(\mathrm{C}_{13} \mathrm{H}_{15} \mathrm{FO}_{4} \mathrm{Na}\right)$ : 277.0847, found: 277.0844 .

\section{Ethyl 2-hydroxy-4-(2-hydroxy-5-methylphenyl)pent-4-enoate} (9h)

Colorless oil, $135 \mathrm{mg}, 54 \%$; ${ }^{1} \mathrm{H}$ NMR (300 MHz, Chloroform- $d, \delta$ ppm) 6.98 (ddd, $J=8.2,2.2,0.7 \mathrm{~Hz}, 1 \mathrm{H}), 6.89-6.87(\mathrm{~m}, 1 \mathrm{H})$, $6.81(\mathrm{~d}, J=8.2 \mathrm{~Hz}, 1 \mathrm{H}$ ), 6.24 ( br s, $1 \mathrm{H}$ ), 5.42 (ddd, $J=1.7,0.9$, $0.8 \mathrm{~Hz}, 1 \mathrm{H}), 5.24(\mathrm{dd}, J=1.7,0.8 \mathrm{~Hz}, 1 \mathrm{H}), 4.27-4.05(\mathrm{~m}, 3 \mathrm{H})$, $3.13(\mathrm{~d}, J=4.9 \mathrm{~Hz}, 1 \mathrm{H}$ ), 2.94 (dddd, $J=14.1,4.6,1.5,0.8 \mathrm{~Hz}$, $1 \mathrm{H}$ ), 2.73 (ddd, $J=14.1,8.6,0.9 \mathrm{~Hz}, 1 \mathrm{H}), 2.26(\mathrm{~s}, 3 \mathrm{H}), 1.26(\mathrm{t}, J=$ $7.1 \mathrm{~Hz}, 3 \mathrm{H}) .{ }^{13} \mathrm{C}$ NMR (101 MHz, Chloroform-d, $\left.\delta \mathrm{ppm}\right){ }^{13} \mathrm{C}$ NMR $\left(101 \mathrm{MHz}, \mathrm{CDCl}_{3}\right) \delta 170.0,160.9,154.1,131.3,124.5,115.6$, 
113.0, 107.4, 101.6, 73.7, 61.4, 55.4, 18.0, 14.2. HRMS (ESI ${ }^{+}$ calculated for $[\mathrm{M}+\mathrm{Na}]^{+}\left(\mathrm{C}_{14} \mathrm{H}_{18} \mathrm{O}_{4} \mathrm{Na}\right)$ : 273.1097, found 273.1098

\section{General procedure for the intramolecular Mitsunobu reaction}

To a solution of phenol 9 (1 mmol) in dry dichloromethane (10 $\mathrm{mL}$ ) was added under argon triphenylphosphine $(262 \mathrm{mg}, 1$ $\mathrm{mmol}$ ) and diisopropyl azodicarboxylate (202 mg, $196 \mu \mathrm{L}, 1$ $\mathrm{mmol}$ ) diluted in $2 \mathrm{~mL}$ of dry DCM. The reaction mixture was stirred overnight. After evaporation of the solvent, the crude residue was purified by column chromatography on silica gel (cyclohexane/AcOEt: 10/1 to 7/3).

\section{Ethyl 4-methylenechromane-2-carboxylate (1a)}

Colorless oil, 172 mg, 79\%; ${ }^{1} \mathrm{H}$ NMR (300 MHz, Chloroform- $d, \delta$ ppm) 7.53 (dd, $J=7.9,1.6 \mathrm{~Hz}, 1 \mathrm{H}$ ), 7.20 (ddd, $J=8.6,7.2,1.6$ $\mathrm{Hz}, 1 \mathrm{H}$ ), 6.98 (dd, $J=8.3,1.2 \mathrm{~Hz}, 1 \mathrm{H}$ ), 6.92 (ddd, $J=7.9,7.2,1.3$ $\mathrm{Hz}, 1 \mathrm{H}), 5.56(\mathrm{~d}, J=1.3 \mathrm{~Hz}, 1 \mathrm{H}), 4.97(\mathrm{~d}, J=1.3 \mathrm{~Hz}, 1 \mathrm{H}), 4.79$ (dd, $J=7.6,4.3 \mathrm{~Hz}, 1 \mathrm{H}), 4.24(\mathrm{q}, J=7.1 \mathrm{~Hz}, 2 \mathrm{H}), 2.95$ (ddt, $J=$ 14.5, 4.3, 1.2 Hz, 1H), 2.87 (ddt, $J=14.9,7.8,1.5 \mathrm{~Hz}, 1 \mathrm{H}$ ), 1.27 $(\mathrm{t}, J=7.1 \mathrm{~Hz}, 3 \mathrm{H}) .{ }^{13} \mathrm{C}$ NMR $(101 \mathrm{MHz}$, Chloroform- $d, \delta \mathrm{ppm})$ $170.1,153.1,134.7,129.8,124.2,121.24,121.20,117.4,108.8$, 74.2, 61.4, 33.6, 14.1. HRMS $\left(\mathrm{ESI}^{+}\right)$calculated for $[\mathrm{M}]^{+}$ $\left(\mathrm{C}_{13} \mathrm{H}_{15} \mathrm{O}_{3}\right): 218.0937$, found: 218.0936 .

\section{Ethyl 7-fluoro-4-methylenechromane-2-carboxylate (1b)}

Colorless oil, $156 \mathrm{mg}, 66 \%$; ${ }^{1} \mathrm{H}$ NMR (300 MHz, Chloroform- $d, \delta$ ppm) 7.47 (dd, $J=8.7,6.4 \mathrm{~Hz}, 1 \mathrm{H}), 6.71-6.60(\mathrm{~m}, 2 \mathrm{H}), 5.47-5.44$ $(\mathrm{m}, 1 \mathrm{H}), 4.94-4.90(\mathrm{~m}, 1 \mathrm{H}), 4.79(\mathrm{dd}, J=7.0,4.5 \mathrm{~Hz}, 1 \mathrm{H}), 4.22$ (q, $J=7.1 \mathrm{~Hz}, 2 \mathrm{H}$ ), $2.92(\mathrm{ddt}, J=14.5,4.4,1.3 \mathrm{~Hz}, 1 \mathrm{H}$ ), 2.84 (ddt, $J=14.5,7.0,1.3 \mathrm{~Hz}, 1 \mathrm{H}), 1.26(\mathrm{t}, J=7.1 \mathrm{~Hz}, 3 \mathrm{H}) .{ }^{13} \mathrm{C} \mathrm{NMR}$ (75 MHz, Chloroform- $d, \delta$ ppm) 169.9, 163.6 (d, $J=247.4 \mathrm{~Hz}$ ), $154.3(\mathrm{~d}, J=12.1 \mathrm{~Hz}), 133.9(\mathrm{~d}, J=1.3 \mathrm{~Hz}), 125.8(\mathrm{~d}, J=9.8 \mathrm{~Hz})$ $117.8(d, J=3.3 \mathrm{~Hz}), 109.0(\mathrm{~d}, J=22.2 \mathrm{~Hz}), 108.7(\mathrm{~d}, J=2.1 \mathrm{~Hz}$ ) $104.5(\mathrm{~d}, J=24.7 \mathrm{~Hz}), 74.5,61.6,33.5,14.2 .{ }^{19} \mathrm{~F} \mathrm{NMR}(282 \mathrm{MHz}$ Chloroform-d, $\delta \mathrm{ppm})$-111.2. HRMS $\left(\mathrm{ESI}^{+}\right)$calculated for [M+Na] ${ }^{+}\left(\mathrm{C}_{13} \mathrm{H}_{13} \mathrm{FO}_{3} \mathrm{Na}\right): 259.0741$, found: 259.0741 .

\section{Ethyl 7-methoxy-4-methylenechromane-2-carboxylate (1c)}

Colorless oil, $198 \mathrm{mg}, 80 \%$ (mixture $1 \mathrm{c}$ and $10 \mathrm{c}$ ); ${ }^{1} \mathrm{H}$ NMR (300 $\mathrm{MHz}$, Chloroform- $d, \delta \mathrm{ppm}) 7.43$ (dd, $J=7.0,2.6 \mathrm{~Hz} 1 \mathrm{H}$ ), 6.54 $(\mathrm{d}, J=2.6 \mathrm{~Hz}, 1 \mathrm{H}), 6.52(\mathrm{~d}, J=7.0 \mathrm{~Hz}, 1 \mathrm{H}), 5.40(\mathrm{~d}, J=0.9 \mathrm{~Hz}$, $1 \mathrm{H}), 4.84(\mathrm{~d}, J=0.6 \mathrm{~Hz}, 1 \mathrm{H}), 4.76(\mathrm{dd}, J=7.8,4.2 \mathrm{~Hz}, 1 \mathrm{H}), 4.23$ $(\mathrm{q}, J=7.1 \mathrm{~Hz}, 2 \mathrm{H}), 3.78(\mathrm{~s}, 3 \mathrm{H}), 2.97-2.78(\mathrm{~m}, 2 \mathrm{H}), 1.28(\mathrm{t}, J=$ $7.2 \mathrm{~Hz}, 3 \mathrm{H})$. These data were extracted from the NMR spectrum of the mixture of the regioisomers by comparison with that of the pure isomer 10c (see below).

\section{Ethyl 7-methoxy-6-methyl-4-methylenechromane-2- carboxylate (1d)}

Colorless oil, $152 \mathrm{mg}, 58 \%$; ${ }^{1} \mathrm{H}$ NMR $(400 \mathrm{MHz}$, Chloroform-d, $\delta$ ppm) $7.27(\mathrm{~s}, 1 \mathrm{H}), 6.47(\mathrm{~s}, 1 \mathrm{H}), 5.38(\mathrm{~s}, 1 \mathrm{H}), 4.82-4.80(\mathrm{~m}, 1 \mathrm{H})$, $4.74(\mathrm{dd}, J=8.2,4.0 \mathrm{~Hz}, 1 \mathrm{H}), 4.25(\mathrm{q}, J=7.1 \mathrm{~Hz}, 2 \mathrm{H}$ ), 3.79 (s, $3 \mathrm{H}$ ), 2.90 (ddt, $J=14.5,3.8,1.2 \mathrm{~Hz}, 1 \mathrm{H}$ ), 2.82 (ddt, $J=14.5,8.2$, $1.2 \mathrm{~Hz}, 1 \mathrm{H})), 2.14(\mathrm{~s}, 3 \mathrm{H}), 1.28(\mathrm{t}, J=7.1 \mathrm{~Hz}, 3 \mathrm{H}) .{ }^{13} \mathrm{C}$ NMR $(101$ $\mathrm{MHz}$, Chloroform- $d, \delta$ ppm) 170.2, 159.1, 152.3, 134.4, 125.5
120.0, 113.0, 106.0, 99.1, 74.4, 61.4, 55.4, 33.9, 15.6, 14.2. HRMS $\left(\mathrm{ESI}^{+}\right)$calculated for $[\mathrm{M}+\mathrm{Na}]^{+}\left(\mathrm{C}_{15} \mathrm{H}_{18} \mathrm{O}_{4} \mathrm{Na}\right)$ : 285.1097, found: 285.1097 .

\section{Ethyl 6-chloro-4-methylenechromane-2-carboxylate. (1f)}

Colorless oil, $192 \mathrm{mg}, 76 \%$; ${ }^{1} \mathrm{H}$ NMR (400 MHz, Chloroform- $d$ ) $\delta$ $7.48(\mathrm{~d}, J=2.5 \mathrm{~Hz}, 1 \mathrm{H}), 7.15$ (dd, $J=8.8,2.5 \mathrm{~Hz}, 1 \mathrm{H}), 6.91(\mathrm{~d}, J=$ $8.8 \mathrm{~Hz}, 1 \mathrm{H}), 5.54(\mathrm{~s}, 1 \mathrm{H}), 5.01(\mathrm{~s}, 1 \mathrm{H}), 4.81-4.75(\mathrm{~m}, 1 \mathrm{H}), 4.23$ (q, $J=7.1 \mathrm{~Hz}, 2 \mathrm{H}), 2.93(\mathrm{dd}, J=14.7,4.2 \mathrm{~Hz}, 1 \mathrm{H}), 2.85$ (dd, $J=$ 14.7, $7.3 \mathrm{~Hz}, 1 \mathrm{H}), 1.29$ (t, $J=7.2 \mathrm{~Hz}, 3 \mathrm{H}) .{ }^{13} \mathrm{C} N M R(75 \mathrm{MHz}$, Chloroform-d) $\delta 170.0,151.8,133.8,129.8,126.5,124.0$, $122.7,119.0,110.3,74.3,61.7,33.3,14.3$. HRMS calculated for $[\mathrm{M}+\mathrm{H}]^{+}\left(\mathrm{C}_{13} \mathrm{H}_{14} \mathrm{ClO}_{3}\right): 253.0626$, found: 253.0628

\section{Ethyl 8-fluoro-4-methylenechromane-2-carboxylate. (1g)}

Colorless oil, $208 \mathrm{mg}, 88 \%$; ${ }^{1} \mathrm{H}$ NMR (300 MHz, Chloroform- $d$, $\delta$ ppm) 7.31 (dt, $J=8.0,1.4 \mathrm{~Hz}, 1 \mathrm{H}$ ), 7.03 (ddd, $J=10.6,8.1,1.5$ $\mathrm{Hz}, 1 \mathrm{H}), 6.86(\mathrm{td}, J=8.1,5.0 \mathrm{~Hz}, 1 \mathrm{H}), 5.62-5.59(\mathrm{~m}, 1 \mathrm{H}), 5.06-$ $5.04(\mathrm{~m}, 1 \mathrm{H}), 4.92(\mathrm{dd}, J=6.3,4.7 \mathrm{~Hz}, 1 \mathrm{H}), 4.22(\mathrm{q}, J=7.1 \mathrm{~Hz}$, $2 \mathrm{H}$ ), 3.00 (ddt, $J=14.8,4.3,1.5 \mathrm{~Hz}, 1 \mathrm{H}$ ), 2.87 (ddt, $J=14.8,6.3$, $1.5 \mathrm{~Hz}, 1 \mathrm{H}), 1.25(\mathrm{t}, J=7.1 \mathrm{~Hz}, 3 \mathrm{H}) .{ }^{13} \mathrm{C}$ NMR $(101 \mathrm{MHz}$, Chloroform- $d, \delta$ ppm) 169.7, 151.9 (d, $J=245.8 \mathrm{~Hz}), 141.6$ (d, $J$ $=11.3 \mathrm{~Hz}), 133.6(\mathrm{~d}, J=3.4 \mathrm{~Hz}), 123.8(\mathrm{~d}, J=1.8 \mathrm{~Hz}), 120.5(\mathrm{~d}, J$ $=7.3 \mathrm{~Hz}), 119.4(\mathrm{~d}, J=3.8 \mathrm{~Hz}), 115.9(\mathrm{~d}, J=18.0 \mathrm{~Hz}), 110.6$, 74.4, 61.7, 33.3, 14.2. ${ }^{19} \mathrm{~F}$ NMR (282 MHz, Chloroform-d, $\delta$ ppm) -136.1. HRMS $\left(\mathrm{ESI}^{+}\right)$calculated for $[\mathrm{M}+\mathrm{Na}]^{+}$ $\left(\mathrm{C}_{13} \mathrm{H}_{13} \mathrm{O}_{3} \mathrm{FNa}\right)$ : 259.0740, found: 259.0739.

\section{Ethyl 6-methyl-4-methylenechromane-2-carboxylate (1h)}

Colorless oil, $179 \mathrm{mg}, 77 \%$; ${ }^{1} \mathrm{H}$ NMR (400 MHz, Chloroform- $d, \delta$ ppm) 7.33 (dd, $J=2.0,0.9 \mathrm{~Hz}, 1 \mathrm{H}), 7.01(\mathrm{dd}, J=8.4,2.1 \mathrm{~Hz}, 1 \mathrm{H})$, $6.88(\mathrm{~d}, J=8.3 \mathrm{~Hz}, 1 \mathrm{H}), 5.55-5.53(\mathrm{~m}, 1 \mathrm{H}), 4.95-4.93(\mathrm{~m}, 1 \mathrm{H})$, 4.75 (dd, $J=7.8,4.1 \mathrm{~Hz}, 1 \mathrm{H}$ ), 4.24 (q, $J=7.1 \mathrm{~Hz}, 2 \mathrm{H}$ ), 2.93 (ddt, $J=14.6,3.8,1.2 \mathrm{~Hz}, 1 \mathrm{H}), 2.85(\mathrm{ddt}, J=14.6,7.8,1.3 \mathrm{~Hz}, 1 \mathrm{H})$, $2.28(\mathrm{~s}, 3 \mathrm{H}), 1.27(\mathrm{t}, J=7.1 \mathrm{~Hz}, 3 \mathrm{H}) .{ }^{13} \mathrm{C} \mathrm{NMR}(101 \mathrm{MHz}$, Chloroform-d, $\delta$ ppm) 170.2, 151.0, 134.8, 130.7, 130.3, 124.3, $120.8,117.2,108.5,74.2,61.4,33.7,20.6,14.1$. HRMS $\left(\mathrm{ESI}^{+}\right)$ calculated for $[\mathrm{M}+\mathrm{Na}]^{+}\left(\mathrm{C}_{14} \mathrm{H}_{16} \mathrm{O}_{3} \mathrm{Na}\right)$ : 255.0991, found: 255.0995 .

\section{General procedure for the isomerisation of 1 to 10}

Trifluoroacetic acid $(8 \mu \mathrm{L}, 0.1 \mathrm{mmol})$ was added to a solution of 4methylenechromane $1(0.1 \mathrm{mmol})$ in dry chloroform $(0.5 \mathrm{~mL})$. The mixture was kept at room temperature for $16 \mathrm{~h}$. The resultant solution was diluted with methylene chloride $(10 \mathrm{~mL})$, washed with an aqueous solution of sodium bicarbonate $(1 \mathrm{M}, 1 \mathrm{~mL})$ and dried over $\mathrm{MgSO}_{4}$. The solvent was evaporated under reduced pressure. The residue was purified by simple filtration on a small pad of silica gel.

Ethyl 4-methyl-2H-chromene-2-carboxylate (10a) 
Colorless oil, $21 \mathrm{mg}, 95 \%$; ${ }^{1} \mathrm{H}$ NMR (400 MHz, Chloroform- $d, \delta$ ppm) 7.22-7.12 (m, 2H), 6.96-6.88 (m, 2H), 5.65-5.60 (m, $1 \mathrm{H})$, 5.35-5.29 $(\mathrm{m}, 1 \mathrm{H}), 4.29-4.14(\mathrm{~m}, 2 \mathrm{H}), 2.05(\mathrm{t}, J=1.6 \mathrm{~Hz}, 3 \mathrm{H})$, $1.27(\mathrm{t}, J=7.1 \mathrm{~Hz}, 3 \mathrm{H}) .{ }^{13} \mathrm{C}$ NMR $(101 \mathrm{MHz}$, Chloroform- $d, \delta$ ppm) 169.9, 152.9, 131.4, 129.6, 123.7, 122.3, 121.4, 115.9, $115.9,73.5,61.4,17.9,14.1$. HRMS $\left(\mathrm{ESI}^{+}\right)$calculated for $[\mathrm{M}+\mathrm{H}]^{+}$ $\left(\mathrm{C}_{13} \mathrm{H}_{15} \mathrm{O}_{3}\right)$ : 219.1016, found: 219.1012 .

\section{Ethyl 7-fluoro-4-methyl-2H-chromene-2-carboxylate (10b)}

Colorless oil, $22 \mathrm{mg}, 94 \% ;{ }^{1} \mathrm{H}$ NMR (300 MHz, Chloroform-d) $\delta$ $7.13(\mathrm{dd}, J=8.2,6.3 \mathrm{~Hz}, 1 \mathrm{H}), 6.74-6.58(\mathrm{~m}, 2 \mathrm{H}), 5.58(\mathrm{dt}, J=$ $4.2,1.6 \mathrm{~Hz}, 1 \mathrm{H}), 5.44(\mathrm{dt}, J=4.2,1.9 \mathrm{~Hz}, 1 \mathrm{H}), 4.31(\mathrm{q}, J=7.1 \mathrm{~Hz}$, $2 \mathrm{H}), 2.06(\mathrm{t}, J=1.6 \mathrm{~Hz}, 3 \mathrm{H}), 1.33(\mathrm{t}, J=7.1 \mathrm{~Hz}, 3 \mathrm{H}) .{ }^{13} \mathrm{C}$ NMR $(75$ $\left.\mathrm{MHz}, \mathrm{CDCl}_{3}\right) \delta 169.8,163.3(\mathrm{~d}, J=247.2 \mathrm{~Hz}), 154.2(\mathrm{~d}, J=12.3$ $\mathrm{Hz}), 130.9,124.8$ (d, $J=9.9 \mathrm{~Hz}), 118.8,114.6,108.2$ (d, $J=21.7$ $\mathrm{Hz}), 103.8(\mathrm{~d}, J=25.3 \mathrm{~Hz}), 73.7,61.6,18.0,14.1 .{ }^{19} \mathrm{~F}$ NMR $(282$ $\mathrm{MHz}$, Chloroform- $d, \delta \mathrm{ppm})$-111.1. HRMS (ESI+) calculated for $[\mathrm{M}+\mathrm{H}]+\left(\mathrm{C}_{13} \mathrm{H}_{14} \mathrm{O}_{3} \mathrm{~F}\right)$ 237.0922, found: 237.0924.

\section{Ethyl 7-methoxy-4-methyl-2H-chromene-2-carboxylate (10c)}

Colorless oil, $24 \mathrm{mg}, 96 \%$; ${ }^{1 \mathrm{H}} \mathrm{NMR}$ (300 MHz, Chloroform- $d$ ) $\delta$ $7.08(\mathrm{~d}, J=8.4 \mathrm{~Hz}, 1 \mathrm{H}), 6.55(\mathrm{~d}, J=2.5 \mathrm{~Hz}, 1 \mathrm{H}), 6.49(\mathrm{dd}, J=8.4$, $2.6 \mathrm{~Hz}, 1 \mathrm{H}), 5.50(\mathrm{dq}, J=4.4,1.5 \mathrm{~Hz}, 1 \mathrm{H}), 5.32(\mathrm{dq}, J=2.8,1.5$ $\mathrm{Hz}, 2 \mathrm{H}), 4.30-4.18(\mathrm{~m}, 2 \mathrm{H}), 3.81(\mathrm{~s}, 3 \mathrm{H}), 2.04(\mathrm{t}, J=1.5 \mathrm{~Hz}, 3 \mathrm{H})$, $1.30(\mathrm{t}, J=7.1 \mathrm{~Hz}, 3 \mathrm{H}) .{ }^{13} \mathrm{C}$ NMR $\left(101 \mathrm{MHz}, \mathrm{CDCl}_{3}\right) \delta 174.2$, $150.8,141.1,129.6,129.1,129.0,127.4,119.8,116.0,68.9$, $62.0,42.2,20.4,14.1$.

\section{Ethyl 7-methoxy-6-methyl-4-methyl-2H-chromene-2 carboxylate (10d)}

Colorless oil, $23 \mathrm{mg}, 88 \%$; RMN ${ }^{1} \mathrm{H}(400 \mathrm{MHz}$, Chloroform- $d, \delta$ ppm) $6.92(\mathrm{~s}, 1 \mathrm{H}), 6.52(\mathrm{~s}, 1 \mathrm{H}), 5.48(\mathrm{dt}, J=4.4,1.5 \mathrm{~Hz}, 1 \mathrm{H})$, $5.30(\mathrm{~d}, J=4.3,1.4 \mathrm{~Hz}, 1 \mathrm{H}).), 4.29-4.15(\mathrm{~m}, 2 \mathrm{H}), 3.83(\mathrm{~s}, 3 \mathrm{H})$, $2.15(\mathrm{~s}, 3 \mathrm{H}), 2.03(\mathrm{t}, J=1.6 \mathrm{~Hz}, 3 \mathrm{H}), 1.30(\mathrm{t}, J=7.1 \mathrm{~Hz}, 3 \mathrm{H})$. $\mathrm{RMN}^{13} \mathrm{C}(101 \mathrm{MHz}$, Chloroform-d, $\delta \mathrm{ppm})$ 170.4, 158. 8, 152.4, $131.7,125.6,119.2,114.77,112.8,99.0,73.8,61.5,55.6,18.1$, 15.7, 14.3. HRMS $\left(\mathrm{ESI}^{+}\right)$calculated for $[\mathrm{M}+\mathrm{Na}]^{+}\left(\mathrm{C}_{15} \mathrm{H}_{18} \mathrm{O}_{4} \mathrm{Na}\right)$ : 285.1097, found: 285.1097 .

Hydrogenation of ethyl 4-methylenechromane-2-carboxylate (1a): access to ethyl 4-methylchromane-2-carboxylate (11a)

A solution of compound 1a (22 mg, $0.1 \mathrm{mmol}$ ) in methanol (5 $\mathrm{mL}$ ) was stirred under hydrogen (1 atm.) in the presence of $5 \%$ $\mathrm{Pd} / \mathrm{C}$ ( 1 mass equiv., $22 \mathrm{mg}$ ) for $16 \mathrm{~h}$ at room temperature. The catalyst was removed by filtration on Celite and the filtrate was evaporated under reduced pressure to give 11 a as a mixture of two diastereomers (87/13).

Colorless oil, $20 \mathrm{mg}, 92 \%$; major isomer: ${ }^{1} \mathrm{H}$ NMR (400 MHz, Chloroform-d, $\delta$ ppm) 7.23 (d, $J=7.7 \mathrm{~Hz}, 1 \mathrm{H}), 7.16(\mathrm{t}, J=7.9$ $\mathrm{Hz}, 1 \mathrm{H}), 7.00-6.89(\mathrm{~m}, 2 \mathrm{H}), 4.67(\mathrm{dd}, J=11.1,2.6 \mathrm{~Hz}, 1 \mathrm{H}), 4.31$ (q, $J=7.1 \mathrm{~Hz}, 2 \mathrm{H}$ ), 3.17-3.06 (m, 1H), 2.40 (ddd, $J=13.4,5.9$, $2.6 \mathrm{~Hz}, 1 \mathrm{H}), 1.85(\mathrm{dt}, J=13.5,11.0 \mathrm{~Hz}, 1 \mathrm{H}), 1.38(\mathrm{~d}, 6.8 \mathrm{~Hz}, 3 \mathrm{H})$, $1.35(\mathrm{t}, J=7.1 \mathrm{~Hz}, 3 \mathrm{H}) .{ }^{13} \mathrm{C}$ NMR $(75 \mathrm{MHz}$, Chloroform- $d, \delta$ ppm) 170.7, 153.2, 127.5, 127.1, 126.7, 121.1, 117.1, 74.1,
61.4, 34.2, 29.1, 20.3, 14.2. HRMS $\left(\mathrm{ESI}^{+}\right)$calculated for [M+Na] ${ }^{+}\left(\mathrm{C}_{13} \mathrm{H}_{16} \mathrm{O}_{3} \mathrm{Na}\right): 243.0991$, found: 243.0993.

Synthesis of ethyl 4-(2-azidophenyl)-2-hydroxypent-4-enoate (12a)

$\mathrm{NaN}_{3}(107 \mathrm{mg}, 1.6 \mathrm{mmol})$ and $\mathrm{Cu}(\mathrm{OAc})_{2}(145 \mathrm{mg}, 0.8 \mathrm{mmol})$ were added to the pinacol boronate $7 \mathrm{a}(173 \mathrm{mg}, 0.8 \mathrm{mmol})$ in $\mathrm{MeOH}(5 \mathrm{~mL})$. The solution was stirred vigorously at reflux for 5 hours. The mixture was concentrated and then extracted with dichloromethane. After evaporation of the solvent, the residue was purified by chromatography on silica gel (cyclohexane/ethyl acetate: 4/1).

Colorless oil, $73 \mathrm{mg}, 55 \%$; ${ }^{1} \mathrm{H}$ NMR (300 MHz, Chloroform- $d, \delta$ ppm) 7.33 (ddd, $J=8.0,7.3,1.7 \mathrm{~Hz}, 1 \mathrm{H}$ ), 7.21 (dd, $J=7.6,1.7$ $\mathrm{Hz}, 1 \mathrm{H}), 7.18-7.02(\mathrm{~m}, 2 \mathrm{H}), 5.34(\mathrm{q}, J=1.3 \mathrm{~Hz}, 1 \mathrm{H}), 5.14$ (d, $J=$ $1.7 \mathrm{~Hz}, 1 \mathrm{H}$ ), 4.19-3.94 (m, 3H), 3.06 (ddd, $J=14.4,4.4,0.8 \mathrm{~Hz}$, $1 \mathrm{H}), 2.95-2.83(\mathrm{br} \mathrm{s}, 1 \mathrm{H}), 2.86$ (ddd, $J=14.4,7.5,1.0 \mathrm{~Hz}, 1 \mathrm{H})$, $1.22(\mathrm{t}, J=7.2 \mathrm{~Hz}, 3 \mathrm{H}) .{ }^{13} \mathrm{C} \mathrm{NMR}(75 \mathrm{MHz}$, Chloroform- $d, \delta \mathrm{ppm})$ $174.3,142.6,137.1,133.7,130.8,128.83,124.8,119.5,118.2$, 69.0, 61.5, 41.6, 14.1. HRMS $\left(\mathrm{ESI}^{+}\right)$calculated for $[\mathrm{M}+\mathrm{Na}]^{+}$ $\left(\mathrm{C}_{13} \mathrm{H}_{15} \mathrm{~N}_{3} \mathrm{O}_{3} \mathrm{Na}\right)$ : 284.1006 found: 284.1004 .

Synthesis of ethyl 2-hydroxy-4-(2-((triphenyl-15phosphanylidene)amino) phenyl)pent-4-enoate (13a)

A mixture of 12a (94 mg, $0.36 \mathrm{mmol}$ ) and triphenylphosphine (94 mg, $0.36 \mathrm{mmol}$ ) in THF $\left(2 \mathrm{~mL}\right.$ ) was heated at $60^{\circ} \mathrm{C}$ for $6 \mathrm{~h}$. Distillation of the solvent gave a solid which was washed with pentane/ethyl acetate (95/5).

White solid, $160 \mathrm{mg}, 90 \% ;{ }^{1} \mathrm{H}$ NMR (300 MHz, Chloroform- $d, \delta$ ppm) $\delta$ 7.80-7.70 (m, 6H), 7.56-7.41 (m, 9H), $7.04(\mathrm{dt}, J=7.3$, $2.1 \mathrm{~Hz}, 1 \mathrm{H}), 6.79(\mathrm{td}, J=7.6,1.8 \mathrm{~Hz}, 1 \mathrm{H}), 6.67(\mathrm{dt}, J=7.3,0.6$ $\mathrm{Hz}, 1 \mathrm{H}), 6.47(\mathrm{dt}, J=7.8,1.2 \mathrm{~Hz}, 1 \mathrm{H}), 5.92(\mathrm{br} \mathrm{s}, 1 \mathrm{H}), 5.47$ (s, $1 \mathrm{H}), 5.08$ (dd, $J=2.5,1.0 \mathrm{~Hz}, 1 \mathrm{H}$ ), 4.28 (dd, $J=9.3,3.6 \mathrm{~Hz}, 1 \mathrm{H}$ ), $4.04(q, J=7.1 \mathrm{~Hz}, 2 \mathrm{H}), 3.08$ (ddd, $J=13.4,3.8,1.3 \mathrm{~Hz}, 1 \mathrm{H}$ ), 2.92 (ddd, $J=13.4,9.4,0.7 \mathrm{~Hz}, 1 \mathrm{H}$ ), $1.16(\mathrm{t}, J=7.1 \mathrm{~Hz}, 3 \mathrm{H}) .{ }^{13} \mathrm{C}$ NMR (75 MHz Chloroform-d, $\delta$ ppm) 174.1, 148.3, 145.9, $136.8,136.5,132.9,132.8,132.0,130.9,129.6,129.2,129.2$, $128.9,128.8,127.4,122.1,121.9,118.2,117.9,69.8,60.8$, 44.9, 14.3. ${ }^{31} \mathrm{P}$ NMR (121 MHz, Chloroform-d, $\left.\delta \mathrm{ppm}\right) 7.16$. HRMS $\left(\mathrm{ESI}^{+}\right)$calculated for $[\mathrm{M}+\mathrm{H}]^{+}\left(\mathrm{C}_{31} \mathrm{H}_{31} \mathrm{NO}_{3} \mathrm{P}\right)$ : 496.2036, found: 496.2035 .

\section{Synthesis of ethyl 4-(2-aminophenyl)-2-hydroxypent-4-} enoate (14a)

A solution of 13a $(49 \mathrm{mg}, 0.1 \mathrm{mmmol})$ in THF- $\mathrm{H}_{2} \mathrm{O}(1 \mathrm{~mL} / 0.5$ $\mathrm{mL}$ ) was heated at $70^{\circ} \mathrm{C}$ overnight. The mixture was concentrated and then extracted with dichloromethane. The residue was purified by chromatography on silica gel (cyclohexane/ethyl acetate: 4/1).

Colorless oil, $20 \mathrm{mg}, 84 \%$; ${ }^{1} \mathrm{H}$ NMR (300 MHz, Chloroform- $d, \delta$ ppm) 7.07 (ddd, $J=7.7,7.6,1.5 \mathrm{~Hz}, 1 \mathrm{H}$ ), 7.01 (dd, $J=7.5,1.6$ $\mathrm{Hz}, 1 \mathrm{H}), 6.79-6.67(\mathrm{~m}, 2 \mathrm{H}), 5.40(\mathrm{~s}, 1 \mathrm{H}), 5.21(\mathrm{~d}, J=1.9 \mathrm{~Hz}, 1 \mathrm{H})$, 4.19 (dd, $J=7.9,4.2 \mathrm{~Hz}, 1 \mathrm{H}), 4.15-3.96(\mathrm{~m}, 2 \mathrm{H}), 3.55$ (br s, $3 \mathrm{H})$, 2.92 (ddd, $J=14.1,3.9,0.61 \mathrm{H}$ ), 2.76 (ddd, $J=14.1,7.9,1.0 \mathrm{~Hz}$, $1 \mathrm{H}), 1.22(\mathrm{t}, J=7.2 \mathrm{~Hz}, 3 \mathrm{H}) .{ }^{13} \mathrm{C}$ NMR $(75 \mathrm{MHz}$, Chloroform- $d, \delta$ 
ppm) 174.4, 143.3, 142.5, 128.8, 128.3, 127.5, 118.9, 118.5, 116.0, 68.9, 61.6, 42.6, 14.1. HRMS $\left(\mathrm{ESI}^{+}\right)$calculated for $[\mathrm{M}+\mathrm{Na}]^{+}\left(\mathrm{C}_{13} \mathrm{H}_{17} \mathrm{NO}_{3} \mathrm{Na}\right): 258.1100$, found : 258.1099.

\section{Synthesis of ethyl 2-hydroxy-3-(1H-indol-3-yl)propanoate (15a)}

A round bottom flask was charged with azide $12 \mathrm{a}$ ( $26 \mathrm{mg}, 0.1$ $\mathrm{mmol})$ in acetonitrile $(1 \mathrm{~mL})$. The solution was degasified, then enlightened at $365 \mathrm{~nm}$ (LT5W T8/010 UV Dudexa lamp from Narva) under stirring at room temperature. ${ }^{42}$ After completion of the reaction as monitored by TLC, solvent was removed under vacuum and the residue was directly purified by chromatography on silica gel (cyclohexane/ethyl acetate: 4/1).

Pale yellow oil, $21 \mathrm{mg}, 89 \%$; ${ }^{1} \mathrm{H}$ NMR (300 MHz, Chloroform-d, $\delta$ ppm) 8.07 (br s, 1H), 7.63 (d, $J=5.3 \mathrm{~Hz}, 1 \mathrm{H}), 7.35$ (dd, $J=8.1$, $0.9 \mathrm{~Hz}, 1 \mathrm{H}), 7.19$ (ddd, $J=8.1,7.0,1.4 \mathrm{~Hz}, 1 \mathrm{H}), 7.15-7.08(\mathrm{~m}$, $2 \mathrm{H}), 4.51(\mathrm{t}, J=5.1 \mathrm{~Hz}, 1 \mathrm{H}), 4.27-4.06(\mathrm{~m}, 2 \mathrm{H}), 3.30$ (ddd, $J=$ 14.8, 4.4, 0.9 Hz, 1H), 3.19 (ddd, $J=14.8,6.1,0.7 \mathrm{~Hz}, 1 \mathrm{H}$ ), 2.81 (br s, 1H), $1.24(\mathrm{t}, J=7.2 \mathrm{~Hz}, 3 \mathrm{H}) \cdot{ }^{13} \mathrm{C} N M R(75 \mathrm{MHz}$, Chloroform- $d, \delta$ ppm) 174.4, 136.1, 127.7, 123.1, 122.1, 119.5, 118.9, 111.1, 110.4, 70.8, 61.6, 30.2, 14.1. HRMS $\left(\mathrm{ESI}^{+}\right)$ calculated for $[\mathrm{M}+\mathrm{Na}]^{+}\left(\mathrm{C}_{13} \mathrm{H}_{15} \mathrm{NO}_{3} \mathrm{Na}\right)$ : 256.0944, found: 256.0946 .

\section{Conflicts of interest}

There are no conflicts to declare.

\section{Acknowledgements}

This work was supported by the University of Rennes 1 and the Centre National de la Recherche Scientifique (CNRS). C. B. thanks the Ministère de l'Enseignement Supérieur et de la Recherche (Algeria) for a financial support (PNE program).

\section{Notes and references}

1 For reviews, see: (a) L. Jalili-Baleh, E. Babaei, S. Abdpour, S. Nasir Abbas Bukhari, A. Foroumadi, A. Ramazani, M. Sharifzadeh, M. Abdollahi and M. Khoobi, Eur. J. Med. Chem., 2018, 152, 570. (b) M. Birringer, K. Siems, A. Maxones, J. Frank and S. Lorkowski, RSC Advances 2018, 8, 4803. (c) S. Emami and Z. Ghanbarimasir, Eur. J. Med. Chem., 2015, 93, 539. (d) D. P. Kamat, S. G. Tilve, V. P. Kamat and J. K. Kirtany, Org. Prep. Proc. Int., 2015, 47, 1.

2 R. Comitato, R. Ambraand and F. Virgili, Antioxidants, 2017, 6, 93 and references therein.

3 M. Marketou, Y. Gupta, S. Jain, P. Vardas, Panos Curr. Hypertens. Rep., 2017, 19, 1 and references therein.

4 S. Mazerbourg, S. Kuntz, I. Grillier-Vuissoz, A. Berthe, M. Geoffroy, S. Flament, A. Bordessa and M. Boisbrun, Curr. Top. Med. Chem., 2016, 16, 2115 and references therein.

5 A. C. Berends, P. G. M. Luiten and C. Nyakas, CNS Drug Rev. 2005, 11, 379.

6 R. S. Muthyala, Y. H. Ju, S. Sheng, L. D. Williams, D. R. Doerge, B. S. Katzenellenbogen, W. G.; Helferich and J. A. Katzenellenbogen, Bioorg. Med. Chem., 2004, 12, 1559.
7 R. K. Gara, V. Sundram, S. C. Chauhan and M. Jaggi, Curr. Med. Chem., 2013, 20, 4177.

8 For some significant examples, see: (a) W. Yu, L. Tong, B. Hu, B. Zhong, J. Hao, T. Ji, S. Zan, C. A. Coburn, O. Selyutin, L. Chen, L. Rokosz, S. Agrawal, R. Liu, S. Curry, P. McMonagle, P. Ingravallo, E. Asante-Appiah, S. Chen and J. A. Kozlowski, J. Med. Chem., 2016, 59, 10228. (b) C. Wagner, P. Jurutka and P. Marshall, PCT Int. Appl., WO 2016140979 A1 20160909, 2016. (c) M. Asada, K. Tani, M. Hirobe, S. Higuchi, K. Fuchibe, R. Oikawa, T. Kotani and H. Takano, PCT Int. Appl. WO 2016111347 A1 20160714, 2016. (d) S. Suzuki, T. Okano and Y. Kawasaki, PCT Int. Appl., WO 2015152196 A1 20151008, 2015. (e) S. Suzuki, T. Okano, R. Horiuchi, N. Hareyama, K. Amikura, N. Yamamoto, Y. Yoshizawa, M. Yagi, K. Serizawa and R. Hayashi, Bioorg. Med. Chem. Lett., 2015, 25, 3368. (f) L. Roux, C. Charrier, E. Salomon, M. Ilhan, P. Bisseret and C. Tarnus, Tetrahedron Lett., 2011, 52, 2586. (g) J. Garcia, S. Barluenga, K. Beebe, L. Neckers and N. Winssinger, Chem. Eur. J., 2010, 16, 9767. (h) S.P. Brown, P. Dransfield, X. Du, Z. Fu, J. Houze, X. Jiao, Lai, S., A.-R. Li, J. Liu, Z. Ma, J.C. Medina, V. Pattaropong, W. Shen, M. Vimolratana, Y. Wang, Z. Wang, M. Yu and L. Zhu, PCT Int. Appl., WO 2010045258 A2 20100422, 2010.(i) M. De Lera Ruiz, K.D. McCormick, C.W. Boyce, R.G. Aslanian, Y. Yu, P. Mangiaracina, J. Zheng, M.Y. Berlin, S.L. Ciesla, C.-Y. Huang and B. Liang, PCT Int. Appl., WO 2008100456 A2 20080821, 2008. (j) J. Zheng, K.D. McCormick, J. Chao, C.W. Boyce, R.G. Aslanian and Y., Yu, PCT Int. Appl., WO 2008100459 A1 20080821, 2008.

9 For some selected recent examples of such transformations, see: Wittig: (a) W. J. Teo and S. Ge, Angew. Chem., Int. Ed., 2018, 57, 12935. (b) M. Zhao, J. Chen, H. Yang and L. Zhou, Chem. Eur. J., 2017, 23, 2783. (c) M. Drouin, S. Tremblay and J.-F. Paquin, Org. Biomol. Chem., 2017, 15, 2376. (d) J.-D. Hamel, M. Cloutier and J.-F. Paquin, Org. Lett., 2016, 18, 1852. Tebbe: (e) A. A. Thomas, K. W. Hunt, M. Volgraf, R. J. Watts, X. Liu, G. Vigers, D. Smith, D. Sammond, T. P. Tang, S. P. Rhodes, A. T. Metcalf, K. D. Brown, J. N. Otten, M. Burkard, A. A. Cox, M. K. G. Do, D. Dutcher, S. Rana, R. K. DeLisle, K. Regal, A. D. Wright, R. Groneberg, K. Scearce-Levie, M. Siu, H. E. Purkey, J. P. Lyssikatos and I. W. Gunawardana, J. Med. Chem., 2014, 57, 878-902. Mc Murry: (f) M. I. Ahmad, S. Dixit, R. Konwar, P. G. Vasdev, A. K. Yadav, S. Tripathi, M. M. Gupta, A. Sharma and A. Gupta, Bioorg. Med. Chem. Lett., 2017, 27, 5040. Knoevenagel: (g) Y. Zhou, J. S. Bandar, R. Y. Liu and S. L. Buchwald, J. Am. Chem. Soc., 2018, 140, 606. (h) Y. Gu, P. Hu, C. Ni and X. Tong, J. Am. Chem. Soc., 2015, 137, 6400. (i) Z. Wu, S. D. Laffoon, T. T. Nguyen, J. D. McAlpin and K. L. Hull, Angew. Chem., Int. Ed., 2017, 56, 1371. Torgov: (j) S. Prevost, N. Dupre, M. Leutzsch, Q. Wang, V. Wakchaure and B. List, Angew. Chem., Int. Ed., 2014, 53, 8770.

10 W. Amberg, U. E. W. Lange, M. Ochse, F. Pohlki, B. Behl, A. L. Relo, W. Hornberger, C. Hoft, M. Mezler, J. Sydor, Y. Wang, H. Zhao, J. T. Brewer, J. Dietrich, H. Li, I. Akritopoulou-Zanze, Y. Lao, S. M. Hannick, Y.-Y. Ku and A. Vasudevan, J. Med. Chem., 2018, 61, 7503.

11 (a) J. M. Ketcham, I. Volchkov, T.-Y. Chen, P. M. Blumberg, N. Kedei, N. E. Lewin and M. J. Krische, J. Am. Chem. Soc., 2016, 138, 13415. (b) V. Hornillos, A. W. van Zijl and B. L. Feringa, Chem. Commun., 2012, 48, 3712. (c) J. Garcia, S. Barluenga, K. Beebe, L. Neckers and N. Winssinger, Chem. Eur. J., 2010, 16, 9767.

12 (a) A. Carral-Menoyo, A. Misol, M. Gomez-Redondo, N. Sotomayor and E. Lete, J. Org. Chem. 2019, 84, 2048. (b) T. A. Davis, C. Wang and T. Rovis, Synlett, 2015, 26, 1520. (c) Z. Shi, M. Boultadakis-Arapinis, D. C. Koester and F. Glorius, Chem. Commun., 2014, 50, 2650.

13 (a) M. Durandetti, L. Hardou, R. Lhermet, M. Rouen and J. Maddaluno, Chem. Eur. J., 2011, 17, 12773. (b) F. Le Strat, D. 
C. Harrowven and J. Maddaluno, J. Org. Chem., 2005, 70, 489.

14 (a) D. F. Fernandez, C. A. B. Rodrigues, M. Calvelo, M. Gulias, J. L. Mascarenas and F. Lopez, ACS Catal., 2018, 8, 7397. (b) S. S. Bera, S. Debbarma, S. Jana and M. S. Maji, Adv. Synth. Catal., 2018, 360, 2204. (c) Z. Shi, M. Boultadakis-Arapinis, D. C. Koester and F. Glorius, Chem. Commun., 2014, 50, 2650. (d) I. D. Jurberg, K. Ikeda, D. Antwi-Omane and F. Gagosz, Isr. J. Chem., 2013, 53, 915.

15 J. Barluenga, M. Trincado, E. Rubio and J. M. Gonzalez, J. Am. Chem. Soc., 2004, 126, 3416.

16 J. Wu, Y.-M. Wang, A. Drljevic, V. Rauniyar, R. J. Phipps and F. D. Toste, Proc. Natl. Acad. Sci. U. S. A., 2013, 110, 13729.

17 S. Jammi, J. Maury, J.-S. Suppo, M. P. Bertrand and L. Feray, J. Org. Chem., 2013, 78, 12566.

18 Z. Wu, X. Sun, K. Potter, Y. Cao, L. N. Zakharov and P. R. Blakemore, Angew. Chem., Int. Ed., 2016, 55, 12285.

19 Y.-C. Xiao and C. Moberg, Org. Lett., 2016, 18, 308.

20 M. Kimura, A. Ezoe, M. Mori and Y. Tamaru, J. Am. Chem. Soc., 2005, 127, 2011.

21 T.-J. Hu, G. Zhang, Y.-H. Chen, C.-G. Feng and G.-Q. Lin, J. Am. Chem. Soc., 2016, 138, 2897.

22 B. N. Hemric, K. Shen and Q. Wang, J. Am. Chem. Soc. 2016, 138, 5813.

23 J. W. B. Fyfe, E. Valverde, C. P. Seath, A. R. Kennedy, J. M. Redmond, N. A. Anderson and A. J. B. Watson, Chem. Eur. J., 2015, 21, 8951.

24 E. P. Gillis and M. D. Burke, J. Am. Chem. Soc., 2007, 129, 6716.

25 A. J. Close, P. Kemmitt, M. Roe and J. Spencer, Org. Biomol. Chem., 2016, 14, 6751

26 For reviews on ene-reactions, see: (a) S. A. Babu, K. K. Krishnan, S. M. Ujwaldev and G. Anilkumar, Asian J. Org. Chem., 2018, 7, 1033. (b) P. Saha and A. K. Saikia, Org. Biomol. Chem., 2018, 16, 2820. (c) C. E. Sleet, U. K. Tambar and P. Maity, Tetrahedron, 2017, 73, 4023. (d) T. J. Mueller, $\mathrm{J}$. in Comprehensive Organic Synthesis, ed. G. A. Molander and P. Knochel, Elsevier, Oxford, 2nd edn, 2014, vol. 5, pp 1 65. (e) X. H. Liu, K. Zheng and X. Feng, Synthesis, 2014, 46, 2241.

27 For reviews related to other approaches to homoallylic alcohols, see: (a) P.-S. Wang, M.-L. Shen, L-Z Gong, Synthesis, 2018, 50, 956. (b) D. M. Sedgwick, M. N. Grayson, S. Fustero, P. Barrio, Synthesis, 2018, 50, 1935. (c) C. Diner, K.J. Szabo, J. Am. Chem. Soc., 2017, 139, 2. (d) Q. Tian, G. Zhang, Synthesis, 2016, 48, 4038.

28 (a) L. Eberlin, A. Mace, A. S. Batsanov, B. Carboni and A. Whiting, ChemistrySelect, 2018, 3, 4557-4561. (b) S. Gupta, P. Xie, Y. Xia and D. Lee, Org. Lett., 2017, 19, 5162. (c) P. Susnik and G. Hilt, Organometallics, 2014, 33, 5907. (d) G. Hilt, F. Erver and K. Harms, Org. Lett., 2011, 13, 304. (e) M. Turks, A. K. Lawrence and P. Vogel, Tetrahedron Lett., 2006, 47, 2783. (f) E. C. Hansen and D. Lee, J. Am. Chem. Soc., 2005, 127, 3252

29 Due to lack of time and an insufficient amount of $\mathbf{4 i}$, the reaction has not been tested with this compound.

30 (a) S. Vshyvenko, M. L. Clapson, I. Suzuki, and D. G. Hall, ACS Med. Chem. Lett., 2016, 7, 1097. (b) K. Kunihiro, L. Dumais, G. Lafitte, E. Varvier, L. Tomas and C. S. Harris, Adv. Synth. Catal., 2018, 360, 2757.

31 A $85 \%$ yield was obtained from isolated 7 a that confirms that the modest overall yield came from the ene reaction step

32 For similar isomerisation reactions, see ref $14 \mathrm{~d}$.

33 K. D. Grimes, A. Gupte, C. C. Aldrich, Synthesis 2010, 1441.

34 CCDC 1878844 contains the supplementary crystallographic data for 13a. These data can be obtained free of charge from The Cambridge Crystallographic Data Centre via www.ccdc.cam.ac.uk/data_request/cif.
35 L. Junk and U. Kazmaier, Org. Biomol. Chem., 2016, 14, 2916. 36 K. Chen, Y. Li, Z. Du and Z. Tao, Synth. Comm., 2015, 45, 663.

37 G. Chen, J. Gui, L. Li and J. Liao, Angew. Chem. Int. Ed., 2011, 50, 7681.

38 A. Bunescu, Q. Wang and J. Zhu, Chem. Eur. J., 2014, 20, 14633.

39 N. Zhao, S. Xie, G. Chen and J. Xu, Chem. Eur. J., 2016, 22, 12634.

40 A. J. Close, P. Kemmitt, M. K. Emmerson and J. Spencer, Tetrahedron, 2014, 70, 9125.

41 The use of undried Sc(OTf $)_{3}$ led to the formation of several unidentified secondary products which causes a significant drop in yield. On the other hand, the use of freshly distilled ethyl glyoxylate does not bring any noticeable improvement.

42 G. Marri, F. Justaud, S. Das, R. Grée, Eur. J. Org. Chem., 2019, 56. 